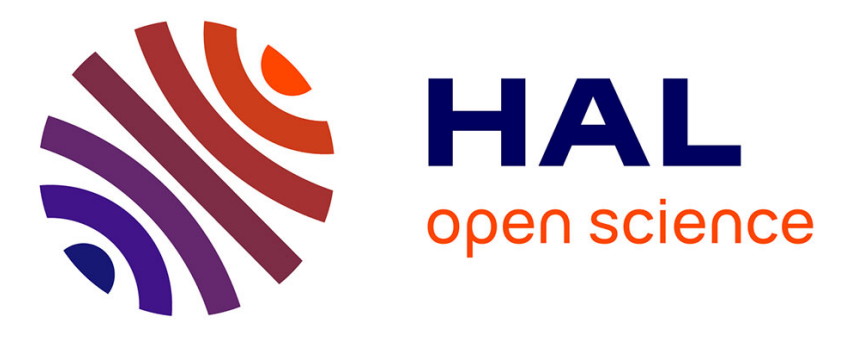

\title{
Optical properties of atomic Mott insulators: From slow light to dynamical Casimir effects
}

\author{
Iacopo Carusotto, Mauro Antezza, Francesco Bariani, Simone de Liberato,
} Cristiano Ciuti

\section{- To cite this version:}

Iacopo Carusotto, Mauro Antezza, Francesco Bariani, Simone de Liberato, Cristiano Ciuti. Optical properties of atomic Mott insulators: From slow light to dynamical Casimir effects. Physical Review A : Atomic, molecular, and optical physics [1990-2015], 2008, 77 (6), pp.063621. 10.1103/PhysRevA.77.063621 . hal-02964258

\section{HAL Id: hal-02964258 \\ https://hal.science/hal-02964258}

Submitted on 12 Oct 2020

HAL is a multi-disciplinary open access archive for the deposit and dissemination of scientific research documents, whether they are published or not. The documents may come from teaching and research institutions in France or abroad, or from public or private research centers.
L'archive ouverte pluridisciplinaire HAL, est destinée au dépôt et à la diffusion de documents scientifiques de niveau recherche, publiés ou non, émanant des établissements d'enseignement et de recherche français ou étrangers, des laboratoires publics ou privés. 


\title{
Optical properties of atomic Mott insulators: From slow light to dynamical Casimir effects
}

\author{
Iacopo Carusotto, ${ }^{1, *}$ Mauro Antezza, ${ }^{1}$ Francesco Bariani, ${ }^{1}$ Simone De Liberato, ${ }^{2,3}$ and Cristiano Ciuti ${ }^{2}$ \\ ${ }^{1}$ CNR-INFM BEC Center and Dipartimento di Fisica, Università di Trento, via Sommarive 14, I-38050 Povo, Italy \\ ${ }^{2}$ Laboratoire Matériaux et Phénomènes Quantiques, Université Paris Diderot-Paris 7 et CNRS, UMR 7162, 75013 Paris, France \\ ${ }^{3}$ Laboratoire Pierre Aigrain, École Normale Supérieure, 24 rue Lhomond, 75005 Paris, France
}

(Received 26 November 2007; published 23 June 2008)

\begin{abstract}
We theoretically study the optical properties of a gas of ultracold, coherently dressed three-level atoms in a Mott insulator phase of an optical lattice. The vacuum state, the band dispersion and the absorption spectrum of the polariton field can be controlled in real time by varying the amplitude and the frequency of the dressing beam. In the weak dressing regime, the system shows unique ultraslow-light propagation properties without absorption. In the presence of a fast time modulation of the dressing amplitude, we predict a significant emission of photon pairs by parametric amplification of the polaritonic zero-point fluctuations. Quantitative considerations on the experimental observability of such a dynamical Casimir effect are presented for the most promising atomic species and level schemes.
\end{abstract}

DOI: 10.1103/PhysRevA.77.063621

PACS number(s): 03.75.Lm, 42.50.Nn, 71.36.+c, 03.70.+k

\section{INTRODUCTION}

Most of the recent advances in the field of nonlinear and quantum optics were made possible by the development of optical media with unprecedented properties. On the one hand, the optical response of carriers in solid-state materials can be controlled and enhanced by confining the carrier motion and/or the photon mode in suitably grown nanostructures [1-5]. On the other hand, systems of ultracold atoms appear as very promising in view of all those applications which require long coherence times, e.g., quantuminformation processing.

Even though the low density of an atomic gas limits the absolute strength of the light-matter coupling, still these systems have the advantage of being almost immune from disorder and decoupled from the environment. Furthermore, they offer the possibility of a precise control and wide tunability of the system parameters in real time by optical and/or magnetic means. In particular, Mott [6,7] (as well as band [8]) insulator states have been realized: Already for a moderately strong lattice potential a few times higher than the atomic recoil energy, a constant and integer number of atoms are trapped in the extremely regular potential of an optical lattice with negligible quantum fluctuations in the atom number. Such systems then constitute an almost perfect realization of the Fano-Hopfield model of resonant dielectrics [9].

In the present paper we present a theoretical study of the classical and quantum optical properties of an atomic Mott insulator. Recently, the case of two-level atoms was investigated in $[10,11]$. Here we extend the Fano-Hopfield model to the case of a three-level system in the presence of a coherent dressing field. The rich potential of three-level configurations has already been demonstrated with the observation of a variety of remarkable effects, such as the quenching of resonant absorption by the so-called electromagnetically induced transparency (EIT) effect $[12,13]$, the light propagation at

\footnotetext{
*carusott@science.unitn.it
}

ultraslow group velocities in the $\mathrm{m} / \mathrm{s}$ range [14,15], and the coherent stopping and storing of light pulses [16]. Here we show how the peculiarities of atomic Mott insulator states can lead to further improvements of these experiments and, even more remarkably, open the way to studies of more subtle quantum optical effects.

Even in its ground state, the electromagnetic (e.m.) field possesses in fact zero-point fluctuations, whose properties are nontrivially affected by the presence of dielectric and/or metallic bodies. One of the most celebrated consequence is the (static) Casimir effect, i.e., the appearance of a force between macroscopic objects due to the zero-point energy of the electromagnetic field $[17,18]$. In the last decades, this force has been the object of intense experimental and theoretical studies in a number of different systems and its main properties can nowadays be considered as reasonably well understood.

The situation is completely different for what concerns the so-called dynamical Casimir effect (DCE) $[19,20]$, i.e., the observable radiation that is emitted by the parametric excitation of the quantum vacuum when the boundary conditions and/or the propagation constants of the electromagnetic field are modulated in time on a very fast time scale. In spite of a wide theoretical literature having addressed this effect for a variety of systems and excitation schemes [21-26], no experimental observation has been reported yet, mainly because of the difficulty of modulating the system parameters at a high enough speed and the presence of competing spurious effects.

In the second part of this paper we show how Mott insulators of coherently dressed three-level atoms are very promising candidates for an experimental observation of the dynamical Casimir effect. As the atomic response to e.m. fields strongly depends on the amplitude and the frequency of the dressing field, a significant time modulation of the optical properties of the atomic Mott insulator can be induced on a very fast time scale by modulating the dressing parameters via standard pulse manipulation techniques [27]. On the other hand, the cleanness of atomic Mott insulator systems allows one to squeeze linewidths down to the spontaneous radiative level and hence to avoid those inhomogeneous 
broadening mechanisms that have so far limited the performances of slow- and stopped-light experiments. Moreover, as the dynamical Casimir radiation is collected in the optical domain and no relaxation processes are involved in the modulation process, no difficulties are expected to appear as a consequence of thermal blackbody radiation or incoherent luminescence from photoexcited carriers [24].

An $a b$ initio model is developed to confirm these expectations in a quantitative way: Taking inspiration from recent works [28-30], we build a microscopic theory of the dynamical Casimir effect in atomic Mott insulators which explicitly includes the matter degrees of freedom. Thanks to the simple form of the resulting parametric Hamiltonian and to the relative weakness of the light-matter coupling constant, expressions for the emission intensity are obtained in closed analytical form. As expected, the most favorable frequency region appears to be the middle polariton branch (the socalled dark-state polariton of [31]), which shows a strong resonant coupling of light with the matter degrees of freedom, as well as a still acceptable amount of absorption losses. Advantages and disadvantages of the atomic Mott insulator system over previously studied solid state systems $[29,30]$ will then be pointed out, as well as the criteria for the choice of the atomic levels to be used. Quantitative estimations of the dynamical Casimir intensity in realistic systems appear as very promising in view of the experimental observation of this still elusive effect. Generalization of the results to experimentally less demanding atomic states, e.g., Bosecondensed clouds or thermal gases is finally discussed.

The structure of the paper is the following. In Sec. II we introduce the physical system and the model used for its theoretical description. In Sec. III we discuss in a systematic way the static properties of the system, such as the dispersion and lifetime of the elementary excitations of the system, the so-called polaritons. A calculation of the dynamical Casimir emission in a spatially infinite, bulk system is presented in Sec. IV, and then extended to experimentally more relevant finite-size geometries in Sec. V. A quantitative discussion of the emission is presented in Sec. VI using realistic parameters of state-of-the-art samples. Conclusions are finally drawn in Sec. VII.

\section{PHYSICAL SYSTEM AND THE THEORETICAL MODEL}

\section{A. The physical system}

We consider a gas of bosonic atoms trapped in the periodic potential of a three-dimensional optical lattice with simple cubic geometry of lattice spacing $a_{L}$. Unless otherwise specified, the system is assumed to be spatially homogeneous with periodic boundary conditions in all three dimensions. The box sizes are equal to $L_{x, y, z}$, and the total volume of the system is $\mathcal{V}=L_{x} L_{y} L_{z}$. For a strong enough lattice potential $V_{0}$ and commensurate filling, the ground state of the system corresponds to a Mott insulator state, with an integer number $n$ of atoms at each lattice site $[6,7]$ and almost negligible fluctuations: The probability of having multiple or zero occupancy of a site decreases in fact proportionally to $\left(a_{L} / a_{s c}\right)^{2} \exp (-4 \sqrt{s})$, where the dimensionless

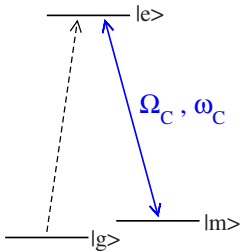

(a) $\Lambda$ scheme

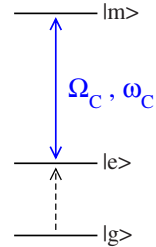

(b) Ladder scheme

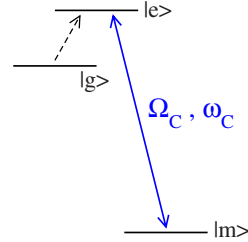

(c) Strongly asymmetric $\Lambda$ scheme
FIG. 1. (Color online) Sketch of the level schemes under consideration.

parameter $s$ is defined as the ratio $s=V_{0} / E_{R}$ of the lattice potential height $V_{0}$ and the atomic recoil energy $E_{R}$ $=\hbar^{2} \pi^{2} / 2 m a_{L}^{2}$ [32], and $a_{s c}$ is the atom-atom scattering length. In what follows we focus our attention on the $n=1$ case in which the atoms are spatially separated and do not interact but via the electromagnetic field. The total number of atoms in the system is thus $N=L_{x} L_{y} L_{z} / a_{L}^{3}$ and the average density $n_{a t}=1 / a_{L}^{3}$. The temperature of the system is assumed to be low enough for the zero temperature approximation to hold. Generalization to the strongly correlated [32] and finite temperature cases is postponed to future work.

The internal atomic dynamics takes place among three internal levels organized in either a $\Lambda$ or a ladder structure [12] as sketched in Figs. 1(a) and 1(b) [the case of a strongly asymmetric $\Lambda$ scheme of Fig. 1(c) will be discussed in Sec. VI]. The atoms are initially prepared in their internal ground state $g$, which is connected to an excited state $e$ by an allowed optical transition of dipole matrix element $d_{e g}$ at a frequency $\omega_{e}$. For notational simplicity, the energy zero is set in a way to have $\omega_{g}=0$. A coupling laser of frequency $\omega_{C}$ dresses the atoms by driving the transition between the excited $e$ level and a third, initially empty, state $m$ of energy $\omega_{m}$. In the $\Lambda$ case, the lifetime of the $m$ state can be very long, much longer than the free-space radiative lifetime of the $e$ state.

In terms of the local amplitude $E_{C}(\mathbf{R})$ of the dressing electric field at the atomic position $\mathbf{R}$, the (complex) Rabi frequency of the coupling is $\hbar \Omega_{C}(\mathbf{R})=d_{e m} E_{C}(\mathbf{R})$. In the following we consider the case where $\Omega_{C}$ is spatially uniform [33]; the discussion of more complex cases is postponed to future works. The direct transition $g \rightarrow m$ is assumed to be optically inactive $d_{g m}=0$.

Provided the lattice potential is strong enough to fulfill the Lamb-Dicke condition and has the same effect on the atoms irrespectively of their internal state, the external degrees of freedom can be decoupled from the internal dynamics and remain frozen in the motional ground state of each lattice site $[34,35]$.

\section{B. The Three-level Fano-Hopfield model}

A quantitative description of the many-atom system interacting with the electromagnetic field can be developed by generalizing the Fano-Hopfield model of a resonant dielectric [9] to the present case of three-level atoms. In this approach, both the atomic electric dipole polarization and the radiation field are described as a collection of coupled har- 
monic oscillators. Neglecting for simplicity all higher-lying photonic bands and assuming the different light polarization to be decoupled, the vector potential operator has a simple "scalar" expression in terms of the photon $(p h)$ creation and annihilation operators $\hat{a}_{p h, \mathbf{k}}^{\dagger}$ and $\hat{a}_{p h, \mathbf{k}}$,

$$
\hat{A}(\mathbf{R})=\sum_{\mathbf{k} \in \mathrm{FBZ}} \sqrt{\frac{2 \pi c \hbar}{k \mathcal{V}}}\left(\hat{a}_{p h, \mathbf{k}} e^{i \mathbf{k} \cdot \mathbf{R}}+\hat{a}_{p h, \mathbf{k}}^{\dagger} e^{-i \mathbf{k} \cdot \mathbf{R}}\right),
$$

where the sum over $\mathbf{k}$ vectors is limited to the first Brillouin zone (FBZ) of the lattice. This approximation holds under the assumption of an isotropic atomic response and in the limit of a small lattice spacing $\omega_{e} a_{L} / c \ll 1$ [51].

In our specific case of three-level atoms, two material degrees of freedom are associated to each atom, which correspond to its excitation from the $g$ state to, respectively, the $e$ and $m$ states. As usual, raising and lowering operators for the excited $e$ state of the $j$ atom are defined as $\hat{a}_{e, j}^{\dagger}|g\rangle_{j}=|e\rangle_{j}$ and $\hat{a}_{e, j}|e\rangle_{j}=|g\rangle_{j}$, and analogously the $\hat{a}_{m, j}^{\dagger}$ and $\hat{a}_{m, j}$ for the $m$ state. In the spirit of the harmonic oscillator model of [9], these operators can be extended as creation and annihilation operators satisfying the usual Bose commutation rules. This bosonic description is accurate under the assumption that the probability for a given atom to be in an excited state is small: In this limit the higher-lying atomic states are not involved in the physics under consideration and the dynamics is restricted to the subspace spanned by the three $|(g, e, m)\rangle$ states for which the harmonic oscillator description is exact. Generally speaking, this assumption is expected to be accurate as long as the number of excitations present in the system is much smaller than the number of atoms [52].

Reabsorbing the phase of the dressing field $\Omega_{C}$ and its time dependence at $\omega_{C}$ into the definition of the $\hat{a}_{m, j}$ and $\hat{a}_{m, j}^{\dagger}$ operators, the internal dynamics of the $j$ atom is described by the following time-independent Hamiltonian:

$$
H_{a t}^{j}=\hbar \omega_{e} \hat{a}_{e, j}^{\dagger} \hat{a}_{e, j}+\hbar \widetilde{\omega}_{m} \hat{a}_{m, j}^{\dagger} \hat{a}_{m, j}+\hbar \Omega_{C}\left(\hat{a}_{e, j}^{\dagger} \hat{a}_{m, j}+\hat{a}_{m, j}^{\dagger} \hat{a}_{e, j}\right)
$$

with a real $\Omega_{C}$ and a renormalized $\widetilde{\omega}_{m}=\omega_{m} \pm \omega_{C}$, the \pm signs referring to, respectively, the $\Lambda$ and the ladder configuration (see Fig. 1).

The bosonic Hamiltonian (2) can be rewritten in terms of the position and momentum operators of two fictitious $e, m$ particles of mass $M$ harmonically bound at frequencies, respectively, $\omega_{e}$ and $\widetilde{\omega}_{m}$, and mechanically coupled by the $\Omega_{C}$ dressing field

$$
\begin{aligned}
H_{a t}^{j}= & \frac{M \omega_{e}^{2}}{2} \hat{X}_{e, j}^{2}+\frac{1}{2 M} \hat{P}_{e, j}^{2}+\frac{M \widetilde{\omega}_{m}^{2}}{2} \hat{X}_{m, j}^{2}+\frac{1}{2 M} \hat{P}_{m, j}^{2} \\
& +M \Omega_{C} \sqrt{\omega_{e} \widetilde{\omega}_{m}} \hat{X}_{e, j} \hat{X}_{m, j}+\frac{\Omega_{C}}{M \sqrt{\omega_{e} \widetilde{\omega}_{m}}} \hat{P}_{e, j} \hat{P}_{m, j} ;
\end{aligned}
$$

the position and momentum operators are defined as usual as

$$
\begin{gathered}
\hat{X}_{e, j}=\sqrt{\frac{\hbar}{2 M \omega_{e}}}\left(\hat{a}_{e, j}+\hat{a}_{e, j}^{\dagger}\right) \\
\hat{P}_{e, j}=i \sqrt{\frac{\hbar M \omega_{e}}{2}}\left(\hat{a}_{e, j}^{\dagger}-\hat{a}_{e, j}\right),
\end{gathered}
$$

$$
\begin{gathered}
\hat{X}_{m, j}=\sqrt{\frac{\hbar}{2 M \widetilde{\omega}_{m}}}\left(\hat{a}_{m, j}+\hat{a}_{m, j}^{\dagger}\right), \\
\hat{P}_{m, j}=i \sqrt{\frac{\hbar M \widetilde{\omega}_{m}}{2}}\left(\hat{a}_{m, j}^{\dagger}-\hat{a}_{m, j}\right) .
\end{gathered}
$$

The electric-dipole coupling of the $g \rightarrow e$ transition to the transverse electromagnetic field [53] is included by giving a charge $q$ to the fictitious particle $e$ and then performing the standard minimal coupling replacement $\hat{P}_{e, j} \rightarrow \hat{P}_{e, j}$ $-q \hat{A}\left(\mathbf{R}_{j}\right) / c$ in the Hamiltonian (3); the vector potential $\hat{A}$ is evaluated by Eq. (1) at the position $\mathbf{R}_{j}$ of the atom. As the $g \rightarrow m$ transition is not optically active, the $m$ particle must be left neutral, and no minimal coupling replacement must be made on the $\hat{P}_{m, j}$ operator.

The charge $q$ and the mass $M$ of the harmonic oscillator model are to be chosen in such a way that the model correctly reproduces the actual optical properties of the atomic system under examination: to this purpose, it is enough that the electric dipole matrix element between the ground and the first excited state of the harmonic oscillator model be equal to the dipole moment of the actual atomic transition. This imposes the condition $d_{e g}=\sqrt{\hbar q^{2} / 2 M \omega_{e}}$ : In what follows, we shall see that all observable physical quantities are a function of $d_{e g}$ only, and do not separately involve the $q$ and $M$ parameters of the model.

To take full advantage of the translational symmetry of the system, it is useful to introduce the collective atomic operators

$$
\hat{a}_{(e, m), \mathbf{k}}^{\dagger}=\frac{1}{\sqrt{N}} \sum_{j} \hat{a}_{(e, m), j}^{\dagger} e^{i \mathbf{k} \cdot \mathbf{R}_{j}}
$$

which create a delocalized atomic excitation with a wave vector $\mathbf{k}$ belonging to the first Brillouin zone of the lattice. Analogously to their localized counterparts $\hat{a}_{(e, m), j}$ and $\hat{a}_{(e, m), j}^{\dagger}$, the $\hat{a}_{(e, m), \mathbf{k}}$ and $\hat{a}_{(e, m), \mathbf{k}}^{\dagger}$ satisfy Bose commutation rules.

Straightforward manipulations lead to the final form of the total light-matter Hamiltonian,

$$
H=\sum_{\mathbf{k}}\left[H_{p h, \mathbf{k}}+H_{a t, \mathbf{k}}+H_{\mathrm{int}, \mathbf{k}}\right],
$$

where

$$
\begin{gathered}
H_{p h, \mathbf{k}}=\hbar c k\left(\hat{a}_{p h, \mathbf{k}}^{\dagger} \hat{a}_{p h, \mathbf{k}}+\frac{1}{2}\right), \\
H_{a t, \mathbf{k}}=\hbar \omega_{e} \hat{a}_{e, \mathbf{k}}^{\dagger} \hat{a}_{e, \mathbf{k}}+\hbar \widetilde{\omega}_{m} \hat{a}_{m, \mathbf{k}}^{\dagger} \hat{a}_{m, \mathbf{k}}+\hbar \Omega_{C}\left(\hat{a}_{e, \mathbf{k}}^{\dagger} \hat{a}_{m, \mathbf{k}}+\hat{a}_{m, \mathbf{k}}^{\dagger} \hat{a}_{e, \mathbf{k}}\right),
\end{gathered}
$$

$$
\begin{aligned}
H_{\mathrm{int}, \mathbf{k}}= & -i \hbar C_{k}\left(\hat{a}_{e,-\mathbf{k}}^{\dagger}-\hat{a}_{e, \mathbf{k}}\right)\left(\hat{a}_{p h,-\mathbf{k}}+\hat{a}_{p h, \mathbf{k}}^{\dagger}\right) \\
& +\hbar D_{k}\left(\hat{a}_{p h, \mathbf{k}}+\hat{a}_{p h,-\mathbf{k}}^{\dagger}\right)\left(\hat{a}_{p h,-\mathbf{k}}+\hat{a}_{p h, \mathbf{k}}^{\dagger}\right) \\
& +\frac{i \hbar C_{k} \Omega_{C}}{\omega_{e}}\left(\hat{a}_{m, \mathbf{k}}-\hat{a}_{m,-\mathbf{k}}^{\dagger}\right)\left(\hat{a}_{p h,-\mathbf{k}}+\hat{a}_{p h, \mathbf{k}}^{\dagger}\right) .
\end{aligned}
$$

All terms consist of quadratic forms in the creation and an- 
nihilation operators of the electromagnetic or the matter polarization fields. The first term $H_{p h, \mathbf{k}}$ is the free e.m. field Hamiltonian. The second term $H_{a t, \mathbf{k}}$ describes the internal dynamics of the dressed atoms. The three lines of the third term $H_{\text {int,k }}$, respectively, account for (i) the dipole coupling of the $g \rightarrow e$ transition to the e.m. field, (ii) the photon renormalization due to the squared vector potential term, and (iii) a coupling between the photon quantum field and the $m$ excitation as a result of the dressing field [54].

The coupling constant $C_{k}$ is equal to

$$
C_{k}=\sqrt{\frac{2 \pi \omega_{e}^{2} n_{a t}}{\hbar c k}} d_{e g},
$$

and $D_{k}=C_{k}^{2} / \omega_{e}$. As expected, these expressions involve the model parameters $q$ and $M$ only via their physical combination $d_{e g}$.

Introducing the vector $\hat{\alpha}_{\mathbf{k}}$ of bosonic operators

$$
\hat{\alpha}_{\mathbf{k}}=\left(\hat{a}_{p h, \mathbf{k}}, \hat{a}_{e, \mathbf{k}}, \hat{a}_{m, \mathbf{k}}, \hat{a}_{p h,-\mathbf{k}}^{\dagger}, \hat{a}_{e,-\mathbf{k}}^{\dagger}, \hat{a}_{m,-\mathbf{k}}^{\dagger}\right)^{T},
$$

and the Bogoliubov metric $\eta=\operatorname{diag}[1,1,1,-1,-1,-1]$, the Hamiltonian (9) can be recast in a simple matricial form,

$$
H=\frac{\hbar}{2} \sum_{\mathbf{k}} \hat{\alpha}_{\mathbf{k}}^{\dagger} \eta \mathcal{H}_{\mathbf{k}} \hat{\alpha}_{\mathbf{k}}+E_{0}
$$

in terms of a $6 \times 6 \eta$-Hermitian $\left(\mathcal{H}^{\dagger} \eta=\eta \mathcal{H}\right)$ Hamiltonian matrix $\mathcal{H}_{\mathbf{k}}$ of the form

$$
\mathcal{H}_{\mathbf{k}}=\left(\begin{array}{cc}
\mathcal{K}_{\mathbf{k}} & \mathcal{Q}_{\mathbf{k}} \\
-\mathcal{Q}_{\mathbf{k}}^{\dagger} & -\mathcal{K}_{\mathbf{k}}^{T}
\end{array}\right) .
$$

where $\mathcal{K}_{k}$ and $\mathcal{Q}_{k}$ are $3 \times 3$ matrices. The constant $E_{0}$ fixes the energy zero: As it has no consequences in what follows, it will be neglected from now on.

The Hermitian matrix

$$
\mathcal{K}_{\mathbf{k}}=\left(\begin{array}{ccc}
c k+2 D_{k} & i C_{k} & i \Omega_{C} C_{k} / \omega_{e} \\
-i C_{k} & \omega_{e} & \Omega_{C} \\
-i \Omega_{C} C_{k} / \omega_{e} & \Omega_{C} & \widetilde{\omega}_{m}
\end{array}\right)
$$

takes into account the free field, the internal atomic dynamics including the dressing beam, as well as the light-matter interaction terms at the level of the so-called rotating wave approximation (RWA): Whenever a radiative photon is absorbed (emitted), an atomic excitation is created (destroyed) at its place [35].

The symmetric matrix

$$
\mathcal{Q}_{\mathbf{k}}=\left(\begin{array}{ccc}
2 D_{k} & -i C_{k} & -i \Omega_{C} C_{k} / \omega_{e} \\
-i C_{k} & 0 & 0 \\
-i \Omega_{C} C_{k} / \omega_{e} & 0 & 0
\end{array}\right)
$$

corresponds instead to those additional terms which describe anti-RWA, off-shell processes where a photon and an atomic excitation are simultaneously destroyed or created.

The relative importance of the RWA $\mathcal{K}_{\mathbf{k}}$ and the anti-RWA $\mathcal{Q}_{\mathbf{k}}$ terms is quantified by the ratio $\bar{C} / \omega_{e}$ of the radiationmatter coupling strength $\bar{C}=C_{k=\omega_{e} / c}$ and the excitation frequency $\omega_{e}$. For most atomic systems of actual experimental interest, this parameter is generally quite small: As a simple example, consider the $D_{2}$ line of ${ }^{87} \mathrm{Rb}$ atoms at $\lambda_{e}$ $=2 \pi c / \omega_{e} \simeq 780 \mathrm{~nm}$. The electric dipole moment of the transition is $d_{e g} \simeq 4.2 e a_{\mathrm{Bohr}}$ [40] and a typical value of lattice spacing is $a_{L}=300 \mathrm{~nm}$. For a unit filling factor $n=1$, the light-matter coupling parameter is then $\bar{C} / \omega_{e} \simeq 1.7 \times 10^{-4}$. Although the condition $\bar{C} / \omega_{e} \ll 1$ rules out the possibility of observing the so-called ultrastrong coupling regime $[29,30]$ in such dilute atomic systems, the anti-RWA terms in the Hamiltonian can still have interesting observable consequences as we shall see in what follows.

\section{STATIONARY STATE: GROUND STATE AND POLARITON EXCITATIONS}

We begin the study of the optical properties of the system from the simplest case where the dressing parameters $\omega_{C}$ and $\Omega_{C}$ are kept fixed in time. For each value of them, the quadratic structure of the Fano-Hopfield Hamiltonian (15) guarantees that this can be set into the canonical form

$$
H=\sum_{\mathbf{k}, r} \hbar \omega_{r, \mathbf{k}} \hat{p}_{r, \mathbf{k}}^{\dagger} \hat{p}_{r, \mathbf{k}}+E_{0}^{\prime}
$$

by means of a Hopfield-Bogoliubov transformation [9]. As in Eq. (15), the constant $E_{0}^{\prime}$ is the zero-point energy and will be neglected from now on. For each wave vector $\mathbf{k}$, the frequencies $\omega_{r, \mathbf{k}}$ of the elementary excitations are given by the eigenvalues corresponding to the positive-norm (in the $\eta$ metric) eigenvectors of the Hopfield-Bogoliubov matrix

$$
\mathcal{M}_{\mathbf{k}}=\left(\mathcal{H}_{\mathbf{k}}\right)^{T}
$$

In the system under consideration here, the elementary excitations are the lower $(r=\mathrm{LP})$, middle $(r=\mathrm{MP}$, often also called dark-state polariton, e.g., in [31]), and upper ( $r=\mathrm{UP})$ polariton modes. All of these modes are linear superposition of light and matter excitations. The polaritonic annihilation operators $\hat{p}_{r, k}$ can be written in terms of the eigenvectors $\vec{w}_{r, k}$ as

$$
\hat{p}_{r, \mathbf{k}}=\sum_{\lambda=1}^{6} w_{r, \mathbf{k}}^{\lambda} \hat{\alpha}_{\mathbf{k}}^{\lambda} .
$$

The index $\lambda$ runs over the six components of the eigenvector $\vec{w}_{r, k}$ of the Hopfield-Bogoliubov matrix (20) and of the operator vector $\hat{\alpha}_{\mathbf{k}}$ defined in Eq. (14). An analogous expression holds for the creation operators $\hat{p}_{r, \mathbf{k}}^{\dagger}$.

Grouping the $\hat{p}_{r, \mathbf{k}}$ 's in the operator vector

$$
\hat{\pi}_{\mathbf{k}}=\left(\hat{p}_{\mathrm{LP}, \mathbf{k}}, \hat{p}_{\mathrm{MP}, \mathbf{k}}, \hat{p}_{\mathrm{UP}, \mathbf{k}}, \hat{p}_{\mathrm{LP},-\mathbf{k}}^{\dagger}, \hat{p}_{\mathrm{MP},-\mathbf{k}}^{\dagger} \hat{p}_{\mathrm{UP},-\mathbf{k}}^{\dagger}\right)^{T},
$$

the transformation (21) to the polaritonic basis can be cast in the simple matricial form $\hat{\pi}_{\mathbf{k}}=\mathbf{W}_{\mathbf{k}} \hat{\alpha}_{\mathbf{k}}$. The first three lines of $\mathbf{W}_{\mathbf{k}}$ correspond to the $\vec{w}_{r, \mathbf{k}}$ eigenvectors (21).

The orthonormality condition of the eigenvectors in the Bogoliubov metric $\eta$ corresponds to the $\eta$-unitary condition

$$
\mathbf{W}_{\mathbf{k}}^{-1}=\eta \mathbf{W}_{\mathbf{k}}^{\dagger} \eta
$$

and guarantees that the operators $\hat{p}_{q, \mathbf{k}}$ satisfy the standard Bose commutation rules. In the $\hat{\pi}$ basis, the Hamiltonian matrix has the simple diagonal form 


$$
\begin{aligned}
\mathcal{H}_{\mathbf{k}}^{\prime} & =\mathbf{W}_{\mathbf{k}} \mathcal{H}_{\mathbf{k}} \mathbf{W}_{\mathbf{k}}^{-1} \\
& =\operatorname{diag}\left[\omega_{\mathrm{LP}, \mathbf{k}}, \omega_{\mathrm{MP}, \mathbf{k}}, \omega_{\mathrm{UP}, \mathbf{k}},-\omega_{\mathrm{LP}, \mathbf{k}},-\omega_{\mathrm{MP}, \mathbf{k}},-\omega_{\mathrm{UP}, \mathbf{k}}\right]
\end{aligned}
$$

In view of the following developments, it is useful to give the explicit form of the $r=\{\mathrm{LP}, \mathrm{MP}, \mathrm{UP}\}$ polariton operators in terms of the photonic $(p h)$ and matter $(e, m)$ excitation ones,

$$
\begin{aligned}
\hat{p}_{r, \mathbf{k}}= & u_{r, \mathbf{k}}^{p h} \hat{\alpha}_{p h, \mathbf{k}}+u_{r, \mathbf{k}}^{e} \hat{a}_{e, \mathbf{k}}+u_{r, \mathbf{k}}^{m} \hat{a}_{m, \mathbf{k}}+v_{r, \mathbf{k}}^{p h} \hat{a}_{p h,-\mathbf{k}}^{\dagger}+v_{r, \mathbf{k}}^{e} \hat{a}_{e,-\mathbf{k}}^{\dagger} \\
& +v_{r, \mathbf{k}}^{m} \hat{a}_{m,-\mathbf{k}}^{\dagger}
\end{aligned}
$$

as well as the inverse transformation $(j=\{p h, e, m\})$,

$$
\begin{aligned}
\hat{a}_{j, \mathbf{k}}= & u_{\mathrm{LP}, \mathbf{k}}^{j *} \hat{p}_{\mathrm{LP}, \mathbf{k}}+u_{\mathrm{MP}, \mathbf{k}}^{j *} \hat{p}_{\mathrm{MP}, \mathbf{k}}+u_{\mathrm{UP}, \mathbf{k}}^{j *} \hat{p}_{\mathrm{UP}, \mathbf{k}}-v_{\mathrm{LP}, \mathbf{k}}^{j} \hat{p}_{\mathrm{LP},-\mathbf{k}}^{\dagger} \\
& -v_{\mathrm{MP}, \mathbf{k}}^{j} \hat{p}_{\mathrm{MP},-\mathbf{k}}^{\dagger}-v_{\mathrm{UP}, \mathbf{k}}^{j} \hat{p}_{\mathrm{UP},-\mathbf{k}}^{\dagger} .
\end{aligned}
$$

The $u$ and $v$ Hopfield coefficients characterize, respectively, the normal and anomalous weights of the different $p h, e, m$ components of the LP, MP, UP polaritons.

\section{A. Polariton vacuum}

The vacuum state of the system corresponds to the ground state $|G\rangle$ of the Hamiltonian (19), and is defined by the vacuum condition

$$
\hat{p}_{r, \mathbf{k}}|G\rangle=0
$$

for all polariton modes $r=\{\mathrm{LP}, \mathrm{MP}, \mathrm{UP}\}$.

As both annihilation and creation operators are involved in the Bogoliubov transformation (26), the ground state $|G\rangle$ corresponds, in the original $\hat{a}_{(p h, e, m)}$ basis, to a squeezed vacuum state with a nonvanishing expectation value of the photon and atomic excitation numbers:

$$
N_{(p h, e, m)}^{G}=\left\langle G\left|\hat{a}_{(p h, e, m), \mathbf{k}}^{\dagger} \hat{a}_{(p h, e, m), \mathbf{k}}\right| G\right\rangle=\sum_{r=\{\mathrm{LP}, \mathrm{MP}, \mathrm{UP}\}}\left|v_{r, \mathbf{k}}^{(p h, e, m)}\right|^{2} .
$$

As the atomic systems under consideration here are far from the ultrastrong coupling regime $[4,29,30]$, an accurate estimation of $N_{(p h, e, m)}^{G}$ can be obtained by means of a suitable perturbation theory in the light-matter coupling strength $\bar{C} / \omega_{e} \ll 1$. The dressing amplitude $\Omega_{C}$ is assumed to be at most of the order of $\bar{C}$.

A zeroth-order approximation $\vec{w}_{q, \mathbf{k}}^{0}$ of the eigenvector can be obtained by diagonalizing the block diagonal matrix

$$
\mathcal{M}_{\mathbf{k}}^{0}=\left(\begin{array}{cc}
\mathcal{K}_{\mathbf{k}}^{T} & 0 \\
0 & -\mathcal{K}_{\mathbf{k}}
\end{array}\right) \text {. }
$$

This provides expressions for the eigenvalues $\omega_{\lambda, \mathbf{k}}^{0}$ and the eigenvectors $\vec{w}_{\lambda, \mathbf{k}}^{0}$ that are correct upto the zeroth-order in $\bar{C} / \omega_{e}$. The eigenvectors have the form

$$
\begin{gathered}
\vec{w}_{\lambda, \mathbf{k}}^{0}=\left(u_{\lambda, \mathbf{k}}^{p h, 0}, u_{\lambda, \mathbf{k}}^{e, 0}, u_{\lambda, \mathbf{k}}^{m, 0}, 0,0,0\right)^{T} \quad(\lambda=1,2,3), \\
\vec{w}_{\lambda, \mathbf{k}}^{0}=\left(0,0,0, u_{\lambda-3, \mathbf{k}}^{p h, 0 *}, u_{\lambda-3, \mathbf{k}}^{e, 0 *}, u_{\lambda-3, \mathbf{k}}^{m, 0 *}\right)^{T} \quad(\lambda=4,5,6) .
\end{gathered}
$$

Up to this level of approximation the virtual occupation (28) is then rigorously vanishing.
When evaluating the first-order correction, attention must be paid to the nonpositive nature of the $\eta$ metric,

$$
\vec{w}_{\lambda, \mathbf{k}}^{1}=\sum_{\substack{\lambda^{\prime}=\{1, \ldots, 6\} \\ \lambda^{\prime} \neq \lambda}} \varepsilon_{\lambda^{\prime}} \frac{\vec{w}_{\lambda^{\prime}, \mathbf{k}}^{0 \dagger} \eta \delta \mathcal{M}_{k} \vec{w}_{\lambda, \mathbf{k}}^{0}}{\omega_{\lambda, \mathbf{k}}^{0}-\omega_{\lambda^{\prime}, \mathbf{k}}^{0}} \vec{w}_{\lambda^{\prime}, \mathbf{k}}^{0},
$$

where the sign $\varepsilon_{\lambda}$ is +1 for $\lambda=1,2,3$, and -1 for $\lambda$ $=4,5,6$. The perturbation matrix $\delta \mathcal{M}_{k}=\mathcal{M}_{k}-\mathcal{M}_{k}^{0}$ is of order $\bar{C} / \omega_{e}$ and has nonzero entries only in the off-diagonal $3 \times 3$ blocks. Keeping in Eq. (32) only the lowest-order terms in $\bar{C} / \omega_{e}$, one gets to the first-order corrections,

$$
v_{r, \mathbf{k}}^{j, 1}=\sum_{r^{\prime}} \frac{-i C_{k}}{\omega_{r, \mathbf{k}}^{0}+\omega_{r^{\prime}, \mathbf{k}}^{0}}\left(u_{r^{\prime}, \mathbf{k}}^{p h, 0} u_{r, \mathbf{k}}^{e, 0}+u_{r^{\prime}, \mathbf{k}}^{e, 0} u_{r, \mathbf{k}}^{p h, 0}\right) u_{r^{\prime}, \mathbf{k}}^{j, 0 *},
$$

where the sum runs over the three polariton branches $r^{\prime}$ $=\{\mathrm{LP}, \mathrm{MP}, \mathrm{UP}\}$. Note that the off-diagonal terms due to the squared vector potential give a contribution to Eq. (33) whose amplitude is of the order of $D_{k} / \omega_{e}=\left(C_{k} / \omega_{e}\right)^{2}$ and have been therefore neglected. The same for the off-diagonal terms due to the dressing field, whose contribution is of the order of $C_{k} \Omega_{C} / \omega_{e}^{2}$.

The virtual population in the ground state is then of the order of $\left(C_{k} / \omega_{e}\right)^{2}$ : Apart from a small region around $\mathbf{k}=0$ where $C_{k}$ diverges, this population is therefore very small for all polariton branches. This fact provides an a posteriori justification of the use of perturbation theory.

In the most significant resonance region $k \simeq k_{e}=\omega_{e} / c$, the frequency denominator of Eq. (33) can be approximated by $2 \omega_{e}$ in a sort of degenerate polariton approximation, which gives

$$
\begin{gathered}
v_{r, k}^{p h, 1} \simeq-\frac{i \bar{C}}{2 \omega_{e}} u_{r, k}^{e, 0}, \\
v_{r, k}^{e, 1} \simeq-\frac{i \bar{C}}{2 \omega_{e}} u_{r, k}^{p h, 0}, \\
v_{r, k}^{m, 1} \simeq 0 .
\end{gathered}
$$

Compact formulas for the fully resonant point $\omega_{e}=\widetilde{\omega}_{m}, k$ $=k_{e}$ of the MP are immediately obtained by inserting in Eq. (34)-(36) the explicit form of the RWA eigenvectors of the zeroth-order Hopfield-Bogoliubov matrix (29),

$$
\begin{gathered}
u_{\mathrm{MP}, k_{e}}^{p h, 0}=\frac{\Omega_{C}}{\left(\bar{C}^{2}+\Omega_{C}^{2}\right)^{1 / 2}}, \\
u_{\mathrm{MP}, k_{e}}^{e, 0}=0, \\
u_{\mathrm{MP}, k_{e}}^{m, 0}=-\frac{i \bar{C}}{\left(\bar{C}^{2}+\Omega_{C}^{2}\right)^{1 / 2}},
\end{gathered}
$$

which leads to anomalous amplitudes

$$
v_{\mathrm{MP}, k_{e}}^{p h, 1} \simeq v_{\mathrm{MP}, k_{e}}^{m, 1} \simeq 0,
$$



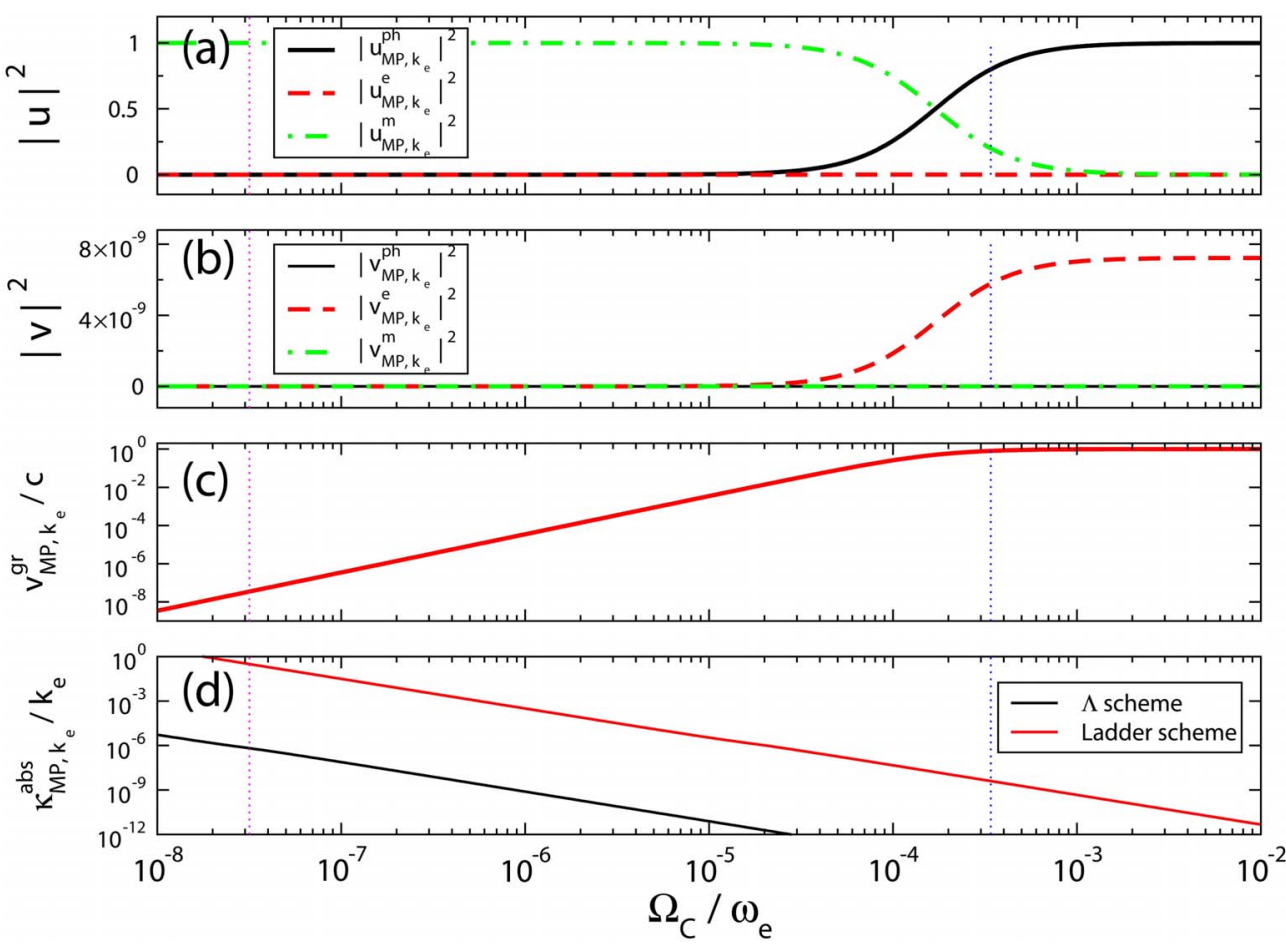

FIG. 2. (Color online) Hopfield $u$ and $v$ coefficients (a,b), group velocity (c), and absorption coefficient (d) of the middle polariton (MP) as a function of dressing amplitude $\Omega_{C}$ in the resonant $\left(\omega_{e}=\widetilde{\omega}_{m}\right)$ case. On the scale of the figure, the analytical approximations (42), (37)-(39), and (41) are undistinguishable from the exact calculations. Vertical lines indicate the dressing amplitude values used in the next figures. The system parameters correspond to the $D_{2}$ line of a Mott insulator system of ${ }^{87} \mathrm{Rb}$ atoms with filling factor $n=1$ in a $a_{L}$ $=300 \mathrm{~nm}$ lattice, $\bar{C} / \omega_{e}=1.7 \times 10^{-4}, \lambda_{e}=2 \pi / k_{e}=2 \pi c / \omega_{e}=780 \mathrm{~nm}$.

$$
v_{\mathrm{MP}, k_{e}}^{e, 1} \simeq-\frac{i \bar{C} \Omega_{C}}{2 \omega_{e}\left(\bar{C}^{2}+\Omega_{C}^{2}\right)^{1 / 2}} .
$$

The accurateness of these analytical approximations is visible in Figs. 2(a) and 2(b) where the Hopfield $u$ and $v$ coefficients are plotted for the fully resonant $k=k_{e}$ point of the MP as a function of the dressing amplitude: On the scale of the figure the analytical approximations are undistinguishable from the exact calculations.

As long as the system parameters are kept constant in time, the virtual populations (28) are intrinsically bound to the system ground state and cannot be revealed by a standard photodetector based on absorption processes [29,30]. On the other hand, the dependence of the anomalous amplitude (41) on $\Omega_{C}$ [see Fig. 2(b)] suggests that the zero-point fluctuations in the ground state can be externally controlled by varying $\Omega_{C}$ in time. In particular, if the time modulation of $\Omega_{C}$ is sufficiently fast, the system is not able to adiabatically follow the time-dependent vacuum state. As a consequence, real polaritons are created in the system and then emitted as radiative photons into the surrounding free space where they can be detected by a photodetector. This will be the subject of Secs. IV-VI.

\section{B. Polariton dispersion}

Examples of polariton dispersion are shown in Fig. 3 for the most significant cases. The shape of the three polariton branches changes in a substantial way depending on the dressing parameters $\omega_{C}$ and $\Omega_{C}$, which provides a simple way to externally vary the optical properties of the system in real time. An application of atomic Mott insulators as dynamic photonic structures was indeed proposed in [36].

As long as linear optical properties are considered, it is important to note that the predictions of the quantum model are indistinguishable from the solution of the Maxwell equations including the semiclassical expression for the local dielectric polarizability of three-level atoms [12]. In particular, this is the case of the band dispersions shown in Fig. 3.

As $\omega_{e}$ and $\widetilde{\omega}_{m}$ have no spatial dispersion, the three bands have no spectral overlap and are separated by energy gaps in which the radiation cannot propagate. For the systems under consideration here, the gaps between the bands are however very narrow, on the order of $\bar{C}^{2} / \omega_{e}$, and therefore almost invisible on the scale of the figure.

When the dressing is on resonance with the $m \rightarrow e$ transition $\left(\omega_{e}=\widetilde{\omega}_{m}\right)$, the atomic resonance is split into a symmetric Autler-Townes doublet at $\omega_{e} \pm \Omega_{C}$ and the photonic mode anticrosses each of the two components. Two subcases are to be distinguished: For a strong dressing $\Omega_{C} \gtrsim \bar{C}$ [panel (c)], the two anticrossings are almost completely separated and have a half-width equal to $\bar{C} / \sqrt{2}$; in between the two anticrossings (i.e., in the neighborhood of $\omega_{e}$ ), the middle polariton (MP) almost coincides with the photon branch and has a group velocity $v_{\mathrm{MP}, k}^{g r}=d \omega_{\mathrm{MP}, k} / d k$ close to $c$. On the other hand, for a weak dressing $\Omega_{C} \ll \bar{C}$, the two anticrossings 

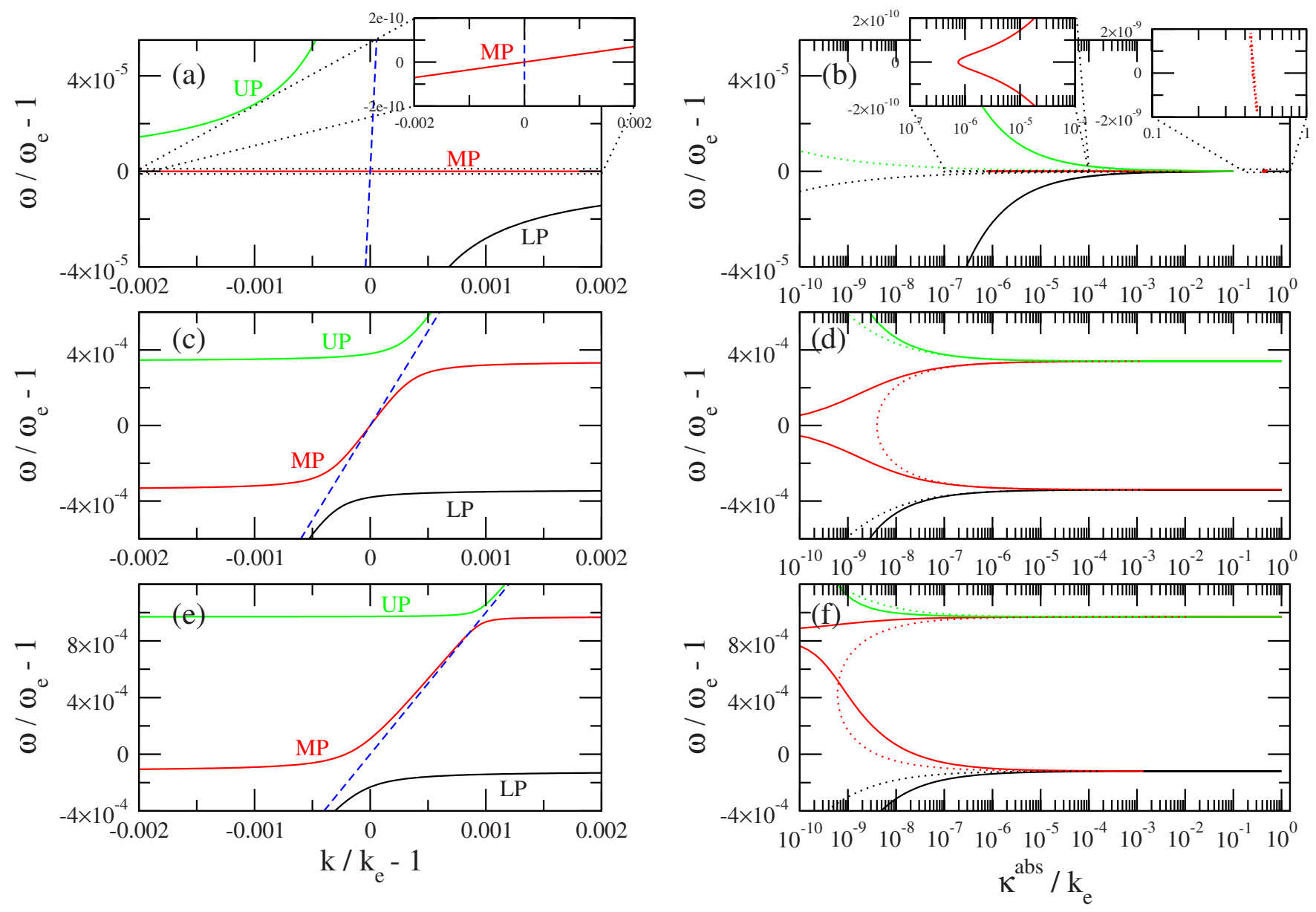

FIG. 3. (Color online) Panels (a,c,e): Dispersion relation $\omega(k)$ of the three polariton modes. Panels (b,d,f): Corresponding absorption spectrum $\kappa^{\text {abs }}$ as a function of the polariton frequency $\omega$. Same system parameters as in Fig. 2. Black, red, and green lines refer to the lower polariton (LP), the middle polariton (MP), the upper polariton (UP), respectively. The blue dashed line in (a,c,e) is the free photon dispersion $\omega=c k$. Panels (a,b): Resonant $\omega_{e}=\widetilde{\omega}_{m}$, weak dressing $\Omega_{C} / \bar{C}=1.86 \times 10^{-4} \ll 1$ case. Insets in (a,b): Magnified views of the most significant parts of the MP branch. Panels (c,d): Resonant $\omega_{e}=\widetilde{\omega}_{m}$, strong dressing $\Omega_{C} / \bar{C}=2$ case. Panels (e,f): Nonresonant $\widetilde{\omega}_{m}-\omega_{e}=5 \bar{C}$, strong dressing $\Omega_{C} / \bar{C}=2$ case. In the absorption (b,d,f) panels: $\Lambda$ level scheme with $\gamma_{e} / 2 \pi=6 \mathrm{MHz}$ and $\gamma_{m} / 2 \pi=10 \mathrm{~Hz}$ (solid lines); ladder level scheme with exchanged $\gamma_{e} / 2 \pi=10 \mathrm{~Hz}$ and $\gamma_{m} / 2 \pi=6 \mathrm{MHz}$ values (dotted lines).

overlap, which results in a strong mutual distortion [panel (a)]: The MP dispersion is strongly flattened, and its group velocity becomes orders of magnitude slower than $c$ [inset of panel (a)].

An approximate, yet quantitatively very accurate expression for the group velocity $v_{\mathrm{MP}, k_{e}}^{g r}$ of the MP around resonance $k=k_{e}=\omega_{e} / c$ is easily obtained from the expression (37) of the MP eigenvector of the RWA Hopfield-Bogoliubov matrix (29),

$$
v_{\mathrm{MP}, k_{e}}^{g r}=c\left|u_{\mathrm{MP}, k_{e}}^{p h, 0}\right|^{2}=c \frac{\Omega_{C}^{2}}{\Omega_{C}^{2}+\bar{C}^{2}} .
$$

As one can see in Fig. 2(c), arbitrarily slow values of $v_{\mathrm{MP}, k_{e}}^{g r}$ can be obtained by simply reducing the amplitude $\Omega_{C}$ of the dressing field: Thanks to the trapping of atoms at lattice sites, no lower bound to the group velocity appears as a consequence of the atomic recoil in absorbing and emitting photons [41]. For the specific case of Rb atoms considered in the figures, a quite conservative value $\Omega_{C} / 2 \pi=12 \mathrm{MHz}$ (i.e., 2 times the radiative lifetime of the $e$ state) already leads to $v_{M, k_{e}}^{g r}=11 \mathrm{~m} / \mathrm{s}$.

In the case of a nonresonant dressing [panel (e)], the oscillator strength of the optical transition is shared in an asymmetric way by the two components of the Autler-Townes doublet. This fact is responsible for the anticrossing to be wider for the component having a larger $e$ state weight (the lower one in the figure). Because of the optical Stark effect, the position of this main anticrossing is slightly shifted by $\Delta \omega_{\mathrm{OSE}}=\Omega_{C}^{2} /\left(\omega_{e}-\widetilde{\omega}_{m}\right)$ from its bare position at $\omega_{e}$.

\section{Effect of losses}

Losses from both the $e$ and the $m$ states are responsible for the finite lifetime of polaritonic excitations. For typical systems, both decay rates $\gamma_{(e, m)}$ are much smaller than the radiation-matter coupling $\bar{C}$ and the energy splitting $\omega_{q, k}$ $-\omega_{q^{\prime}, k}$ between different polariton bands $q \neq q^{\prime}$ at a given 
wave vector $\mathbf{k}$. In optics, this regime goes often under the name of strong coupling regime $[2,3]$ and is characterized by losses not being able to effectively mix the different polariton branches, which retain their individuality.

The Fermi golden rule then provides an accurate prediction for the decay rate $\gamma_{r, \mathbf{k}}$ of the plane-wave polariton state of wave vector $\mathbf{k}$ on the $r$ branch,

$$
\gamma_{r, \mathbf{k}}=\gamma_{e}\left|u_{r, \mathbf{k}}^{e}\right|^{2}+\gamma_{m}\left|u_{r, \mathbf{k}}^{m}\right|^{2}
$$

physically, this decay rate is the relevant one in a light stopping experiment where light is stored in the system during a macroscopically long time [16,31]. In a propagation geometry, a more relevant quantity is instead the absorption length [42],

$$
\ell_{r, \mathbf{k}}=\left(\kappa_{r, \mathbf{k}}^{\mathrm{abs}}\right)^{-1}=v_{r, \mathbf{k}}^{g r} / \gamma_{r, \mathbf{k}} .
$$

The relative value of the $\gamma_{(e, m)}$ loss rates depends on the specific level scheme under consideration.

In a $\Lambda$ configuration, $\gamma_{m}$ is mostly given by nonradiative effects, and generally has a very small value. As no intrinsic effect is expected to significantly contribute to $\gamma_{m}$ in Mott insulator states, it can in principle be suppressed to arbitrarily small values by means of a careful experimental setup.

As a consequence of translational symmetry, energy exchange can only take place between discrete states at the same $\mathbf{k}$, so irreversible radiative decay from the $e$ state to the $g$ state is forbidden. This remarkable fact was discussed at length in the pioneering paper [9], where it was pointed out that polaritons in a rigid lattice of two-level atoms are not subject to dissipation and can propagate with a slow group velocity along macroscopic distances. It is important to note that this fact is strictly related to the Lamb-Dicke freezing of the atomic motion in the ground state of each site, which guarantees, e.g., that atoms cannot decay from the $e$ state into motionally excited states of the $g$ level as instead happens for free atoms in the absence of a lattice [55].

On the other hand, radiative decay from the $e$ state into the $m$ state can occur by spontaneous emission of a photon into a spatial mode different from the one of the coherent dressing field. The contribution of such process to $\gamma_{e}$ is equal to the $e \rightarrow m$ radiative decay rate of an isolated atom in free space [56]. This can only be reduced by choosing a suitably weak $e \leftrightarrow m$ optical transition to dress the system.

In a ladder configuration, the roles of $\gamma_{e}$ and $\gamma_{m}$ are exchanged. Spontaneous emission processes into spatial modes different from the dressing one can now occur for the $m$ $\rightarrow e$ transition, which provides a significant contribution to $\gamma_{m}$. On the other hand, no irreversible radiative decay process on the $e \rightarrow g$ transition can contribute to $\gamma_{e}$ : Provided no other decay channel is available for the $e$ state, a careful design of the experimental setup may then lead to a reduction of $\gamma_{e}$ to arbitrarily small values.

Plots of the prediction (44) for the absorption coefficient for the three polariton branches are shown in Figs. 3(b), 3(d), and 3(f) for, respectively, the $\Lambda$ (solid lines) and the ladder (dashed lines) three-level schemes [57]. Again, these curves are in perfect agreement with the solution of the Maxwell equations using the semiclassical expression for the dielectric polarizability of three-level atoms [12]. In both cases of
$\Lambda$ and ladder configuration, absorption is strongly peaked in the anticrossing regions where polaritonic bands have the largest weight of matter excitations and the slowest group velocity. The only exception is the dip that is visible exactly on resonance with the two-photon Raman transition $\omega=\widetilde{\omega}_{m}$ in the case of a $\Lambda$ configuration. In optics, this effect goes under the name of electromagnetic induced transparency (EIT) effect $[12,13]$ : In the vicinity of the resonance, quantum interference suppresses the weight of $e$ excitation in the MP mode. Consequently, the absorption rate is quenched to the nonradiative one, $\gamma_{\mathrm{MP}, k_{e}} \simeq \gamma_{m} \ll \gamma_{e}$. This effect is most dramatic in the case of a weak dressing $\Omega_{C} \ll \bar{C}$ [see in particular the left-hand inset of Fig. 3(b)], where the minimum of the spatial absorption coefficient $\kappa_{\mathrm{MP}, k_{e}}^{\mathrm{abs}}$ around resonance remains remarkably deep in spite of the very slow group velocity $v_{\mathrm{MP}, k_{e}}^{g r} \ll c$. This allows for MP polaritons to propagate at ultraslow velocities for macroscopic distances without being appreciably absorbed [58]: As one can see in Figs. 2(c) and 2(d), a group velocity as slow as $v_{\mathrm{MP}, k_{e}}^{g r}=15 \mathrm{~m} / \mathrm{s}$ still corresponds to an absorption length as large as $\ell_{\mathrm{MP}, k}$ $\simeq 16 \mathrm{~cm}$.

To conclude this section, it is useful to summarize the main advantages of using a Mott insulator state for slowlight experiments:

(1) Atomic Mott insulators constitute an almost ideal realization of the two-level Fano-Hopfield model [9-11]: Provided one is in the Lamb-Dicke trapping regime, resonant light can propagate at slow group velocities without being absorbed nor scattered as instead happens in homogeneous gases in the absence of the lattice.

(2) The typical features of light propagating in systems of dressed three-level atoms such as EIT and ultraslow group velocities without absorption are further improved. As the atoms interact with each other only via the electromagnetic field, no intrinsic decoherence effect can contribute to the $\gamma_{m}$ rate in a $\Lambda$ configuration nor to the $\gamma_{e}$ one in a ladder configuration.

(3) The presence of a single atom at each lattice site eliminates the inhomogeneous broadening of the transitions that originates e.g., from the spatially varying density profile of a Bose-Einstein condensate.

(4) The trapping of atoms at the lattice sites eliminates the lower bound to the group velocity that atomic recoil would impose in the case of a homogeneous, untrapped gas [41].

\section{DYNAMICAL CASIMIR EMISSION IN THE PRESENCE OF A TIME MODULATION}

When the dressing parameters $\omega_{C}$ and $\Omega_{C}$ are modulated in time, the quadratic form of the Hamiltonian is preserved, yet with a time-dependent Hamiltonian matrix $\mathcal{H}_{\mathbf{k}}(t)=\overline{\mathcal{H}}_{\mathbf{k}}$ $+\delta \mathcal{H}_{\mathbf{k}}(t)$. At each time $t$, a Bogoliubov transformation diagonalizing the instantaneous Hamiltonian can still be found, but the transformation matrix $\mathbf{W}_{\mathbf{k}}(t)$, as well as the polariton bands $\omega_{r, k}$ and the expression of the polariton operators $\hat{p}_{r, \mathbf{k}}$ in terms of the original $\hat{a}_{j, \mathbf{k}}$ ones are now varying in time, as well as the vacuum state $|G(t)\rangle$ of the system. While for slow modulations the system is able to adiabatically follow the 
instantaneous ground state $|G(t)\rangle$, excitations are created in the case of a faster modulation. The study of the properties and the intensity of this dynamical Casimir emission is the subject of the present and the next sections.

Our strategy is to reduce the problem to a simple and tractable parametric Hamiltonian to which the standard tools of quantum optics can be applied. In particular, we shall concentrate our attention on the simplest case of a weak time modulation $\left\|\delta \mathcal{H}_{\mathbf{k}}\right\| \ll\left\|\overline{\mathcal{H}}_{\mathbf{k}}\right\|$ for which perturbation theory can be used to obtain analytical predictions: Most among the perturbations that one can envisage to apply to the atomic system largely fulfill in fact this condition. A discussion of the physics beyond perturbation theory can be found in $[21,25,29,30]$.

Let $\overline{\mathbf{W}}_{\mathbf{k}}$ be the Bogoliubov transformation diagonalizing the unperturbed Hamiltonian matrix $\overline{\mathcal{H}}_{\mathbf{k}}$. In general, the perturbation Hamiltonian

$$
\delta \mathcal{H}_{\mathbf{k}}^{\prime}(t)=\overline{\mathbf{W}}_{\mathbf{k}} \delta \mathcal{H}_{\mathbf{k}}(t) \overline{\mathbf{W}}_{\mathbf{k}}^{-1}
$$

is not diagonal in this basis. In terms of polariton creation and annihilation operators, $\delta \mathcal{H}_{\mathbf{k}}^{\prime}$ introduces terms of two kinds [44]. The diagonal $3 \times 3$ blocks correspond to terms of the form $\hat{p}_{r, \mathbf{k}}^{\dagger} \hat{p}_{s, \mathbf{k}}$, which are responsible for a renormalization of the polariton energies (for $r=s$ ) and for the occurrence of interbranch transitions (for $r \neq s$ ) which transfer already existing polaritons from one branch to another. At the lowest order, these terms have no effect on the polaritonic vacuum state and will therefore not be considered in what follows.

The off-diagonal $3 \times 3$ blocks of $\delta \mathcal{H}_{\mathbf{k}}^{\prime}$ are more interesting in the present context, as they correspond to terms of the forms $\hat{p}_{r, \mathbf{k}}^{\dagger} \hat{p}_{s,-\mathbf{k}}^{\dagger}$ and $\hat{p}_{r, \mathbf{k}} \hat{p}_{s,-\mathbf{k}}$, which, respectively, create or destroy pairs of polaritons in the opposite $\pm \mathbf{k}$ momentum states of the $r$ and $s$ branches $(r, s=\{\mathrm{LP}, \mathrm{MP}, \mathrm{UP}\})$. In particular, they account for the creation of correlated pairs of polaritons out of the vacuum state of the system via parametric amplification of the zero-point quantum fluctuations. This emission of radiation is an example of the still unobserved dynamical Casimir effect (DCE) [19]. The most celebrated example of DCE is predicted for a metallic cavity whose length is varied in time by means of a mechanical motion of its mirrors [21]. Another possibility consists of varying the effective length of the cavity by modulating the mirror conductivity $[23,24]$, by mimicking moving mirrors via a suitably chosen $\chi^{(2)}$ nonlinear optical element [25], or by varying the bulk refractive index of the cavity material $[22,44]$.

A generalization of this latter scheme is the subject of the present paper: The modulation of the dielectric properties of the atomic medium is created by varying in time the dressing field amplitude. Using the polaritonic formalism developed in the previous sections, we will be able to go beyond the nondispersive dielectric approximation made by most of the existing works, so to fully include the resonant dynamics of matter excitations. Using this microscopic model, accurate predictions will be obtained also for the frequency window in the neighborhood of the atomic resonance where the intensity of the dynamical Casimir emission is expected to be the strongest.

\section{A. Polariton emission rate in a bulk geometry}

Depending on the frequency spectrum of the time modulation $\delta \mathcal{H}_{\mathbf{k}}(t)$, polaritons can be emitted in any momentum state: A monochromatic oscillation at a frequency $\omega$ of the form $\delta \mathcal{H}_{\mathbf{k}}(t)=\delta \mathcal{H}_{\mathbf{k}}\left(e^{i \omega t}+e^{-i \omega t}\right)$ is in fact able to resonantly create pairs of polaritons in the $r, s$ branches at wave vectors $\pm \mathbf{k}$ fulfilling the parametric resonance condition

$$
\omega_{r, \mathbf{k}}+\omega_{s,-\mathbf{k}}=\omega .
$$

Note that an analogous condition was recently obtained for the parametric emission of phonons in trapped atomic BoseEinstein condensate in an optical lattice when the lattice potential is modulated in time $[45,46]$.

Starting from the polaritonic vacuum as initial state, and limiting ourselves to lowest-order effects in the modulation amplitude, we can safely neglect the $\hat{p}_{r, \mathbf{k}}^{\dagger} \hat{p}_{s, \mathbf{k}}$ terms that arise from the modulation and rewrite the time-dependent system Hamiltonian (15) in the standard parametric form [47],

$$
H=\bar{H}+\delta H=\bar{H}+\frac{\hbar}{2} \sum_{r s, \mathbf{k}}\left(V_{r s, \mathbf{k}} e^{-i \omega t} \hat{p}_{r, \mathbf{k}}^{\dagger} \hat{p}_{s,-\mathbf{k}}^{\dagger}+\text { H.c. }\right) \text {. }
$$

From this Hamiltonian the polariton creation rate is determined by means of the Fermi golden rule. As final states, one must consider pairs of polaritons created in the $r, s$ $=\{\mathrm{LP}, \mathrm{MP}, \mathrm{UP}\}$ branches at wave vectors $\pm \mathbf{k}$,

$$
\frac{d N_{r s}}{d t}=\frac{2 \pi}{\hbar^{2}} \sum_{\mathbf{k}}|\langle r, \mathbf{k} ; s,-\mathbf{k}|\delta H| G\rangle|^{2} \delta\left(\omega_{r, \mathbf{k}}+\omega_{s,-\mathbf{k}}-\omega\right) .
$$

In the weak modulation regime (i.e., far below any parametric oscillation threshold), this approach is equivalent to other ones based, e.g., on the input-output formalism; including all neglected terms is instead crucial if one is interested in the peculiar pulse shaping and frequency up-conversion effects discussed at length in $[21,30]$.

Replacing as usual the sum over $\mathbf{k}$ vectors with an integral, the total creation rate per unit volume into the $r, s$ branches reads as

$$
\frac{d N_{r s}}{d t d \mathcal{V}}=\frac{\bar{k}^{2}}{2 \pi\left(v_{r, \bar{k}}^{g r}+v_{s, \bar{k}}^{g r}\right)}\left|V_{r s, \bar{k}}\right|^{2},
$$

where the $r(s)$ polariton is assumed to be emitted in the $k_{z}>0\left(k_{z}<0\right)$ half-space. $\bar{k}$ is the wave-vector value at which the resonant condition (46) is satisfied for the $r, s$ branches under examination. $v_{(r, s), \bar{k}}^{g r}$ are the group velocities, and the matrix element $V_{r s, k}$ of the process is given by

$$
V_{r s, k}=\left.\mathbf{W}_{\mathbf{k}} \delta \mathcal{H}_{\mathbf{k}} \overline{\mathbf{W}}_{\mathbf{k}}^{-1}\right|_{r, s+3} ;
$$

here, the $\{\mathrm{LP}, \mathrm{MP}, \mathrm{UP}\}$ branches correspond to, respectively, $r, s=\{1,2,3\}$. Perturbative expressions for the matrix element (50) to the leading order in $\bar{C} / \omega_{e}$ will be given in Sec. IV B.

It is worth noticing that a finite emission intensity $\left\langle\hat{p}_{r, \mathbf{k}}^{\dagger} \hat{p}_{r, \mathbf{k}}\right\rangle \neq 0$ is obtained even though the classical amplitudes remain strictly zero $p_{r, \mathbf{k}}=\left\langle\hat{p}_{r, \mathbf{k}}\right\rangle=0$ during the whole 
modulation process. The dynamical Casimir emission is in fact a purely quantum effect due to the parametric amplification of the zero-point fluctuations of the polariton field.

Equations (49) and (50) are the central result of the present section: They quantify the polariton emission in an idealized, spatially infinite system and will represent the central building block in the study of experimentally relevant finite-size geometries that we shall perform in Sec. V. A quantitative discussion of the actual value of the emission rate for realistic values of the system parameters will be given in Sec. VI.

\section{B. Approximate analytical expression of the matrix element}

A simple, yet accurate estimation of the matrix element (50) can be obtained by means of the perturbative approximation of the eigenvectors $\vec{w}_{r, k}$ discussed in Sec. III A. As a most significant example, we consider a periodic modulation of the coupling amplitude $\Omega_{C}$ of the form

$$
\Omega_{C}(t)=\Omega_{C}+\delta \Omega_{C}\left(e^{-i \omega t}+e^{i \omega t}\right),
$$

while its frequency $\omega_{C}$ is kept constant [59]. Although our theory is completely general, it is interesting to note that in this specific case the dynamical Casimir effect can be interpreted as a peculiar kind of parametric down-conversion. In frequency space, the time-dependent dressing field amplitude (51) consists in fact of a triplet of lines spaced by $\omega$ : The energy $\hbar \omega$ that is available upon absorption from one line and subsequent stimulated reemission into the lower one is parametrically converted into a pair of dynamical Casimir polaritons. Modulations of $\Omega_{C}$ were also at the heart of recent light-stopping experiments [16], albeit with much slower characteristic ramp times: In that case, the modulation had in fact to be adiabatic enough not to induce interbranch transitions.

Inserting the perturbative result (34)-(36) into the $\overline{\mathbf{W}}_{\mathbf{k}}$ transformation matrix, the following expression for the matrix element is found, which is valid throughout the whole resonance region:

$$
V_{r s, k} \simeq-\frac{i \bar{C}}{2 \omega_{e}}\left(u_{r, k}^{p h} u_{s, k}^{m}+u_{s, k}^{p h} u_{r, k}^{m}\right) \delta \Omega_{C}
$$

As expected, $V_{r s, k}$ is proportional to the amplitude of the dressing modulation and to $\bar{C} / 2 \omega_{e}$, i.e., the ratio between the amplitude of the antiresonant light-matter coupling and the energy associated to the creation of a pair of excitations. The presence in Eq. (52) of the Hopfield coefficients of both the photonic and the $m$ excitations means that a mixing between light and matter modes is necessary to obtain a sizeable emission. A most favorable region is therefore the fully resonant point where $\omega_{e}=\widetilde{\omega}_{m}$ and the modulation is driven at a frequency $\omega=2 \omega_{e}$.

An analytical estimation of $V_{r s, k_{e}}$ with $r=s=\mathrm{MP}$ is readily obtained for this case by inserting in Eq. (52) the analytical eigenvector (37)-(39) of the zeroth-order HopfieldBogoliubov matrix (29),

$$
V_{\mathrm{MP}, k_{e}}=\frac{\Omega_{C}}{\omega_{e}} \frac{\bar{C}^{2}}{\bar{C}^{2}+\Omega_{C}^{2}} \delta \Omega_{C} .
$$

In the slow-light regime $\Omega_{C} \ll \bar{C}$, the matrix element $V_{\mathrm{MP}, k_{e}}$ grows as $\Omega_{C} \delta \Omega_{C} / \omega_{e}$, while it goes as $\bar{C}^{2} \delta \Omega_{C} /\left(\Omega_{C} \omega_{e}\right)$ for $\Omega_{C} \gg \bar{C}$.

Using the expression (42) for the group velocity, it is immediate to see that for a given value of the relative modulation amplitude $\delta \Omega_{C} / \Omega_{C}$, the emission intensity (49) starts proportionally to $\Omega_{C}^{2}$ for small values $\Omega_{C} \ll \bar{C}$ and then saturates to a finite value for $\Omega_{C} \gg \bar{C}$. We will come back to these issues in the quantitative discussion of Sec. VI.

The main result (52) of the present section fully includes the dispersion of polaritons, which is crucial to correctly describe the region around resonance. This contrasts to previous works [44] where the Casimir emission was studied for a dispersionless dielectric medium with a periodically oscillating dielectric constant

$$
\boldsymbol{\epsilon}(t)=\bar{\epsilon}+\delta \epsilon\left(e^{i \omega t}+e^{-i \omega t}\right) .
$$

Even in this case, the Hamiltonian of the system can be reduced to the parametric form (47), with the matrix element

$$
V_{k}=\frac{\omega}{4 \bar{\epsilon}} \delta \epsilon
$$

As a single photonic branch is considered, no $r, s$ indices are needed. A quantitative comparison of the two approaches will be made in Sec. VI: Agreement is expected to hold in the low-frequency limit where the dispersion of the dielectric constant can be indeed neglected.

\section{EMISSION RATE FROM FINITE-SIZE SYSTEMS}

In order to obtain a quantitative prediction for an actual experimental setup, one must go beyond the idealized infinite geometry system considered so far, and study the more realistic case where dynamical Casimir light is generated in a spatially finite system and then revealed by a detector located in the external vacuum.

The first step is to rewrite the parametric Hamiltonian (47) for a bulk system in a local form. Expressing the polariton operators $\hat{p}_{r, \mathbf{k}}$ in terms of the real-space ones

$$
\hat{p}_{r, \mathbf{k}}=\frac{1}{\sqrt{\mathcal{V}}} \int d^{3} \mathbf{x} e^{-i \mathbf{k} \cdot \mathbf{x}} \hat{\psi}_{r}(\mathbf{x}),
$$

the parametric Hamiltonian [see Eq. (47)] can be rewritten as

$$
\delta H=\frac{\hbar}{2} \sum_{r s} \int d^{3} \mathbf{x} d^{3} \mathbf{x}^{\prime} \hat{\psi}_{r}^{\dagger}(\mathbf{x}) \hat{\psi}_{s}^{\dagger}\left(\mathbf{x}^{\prime}\right) \tilde{V}_{r s}\left(\mathbf{x}-\mathbf{x}^{\prime}\right) e^{-i \omega t}+\text { H.c. }
$$

Here the kernel 


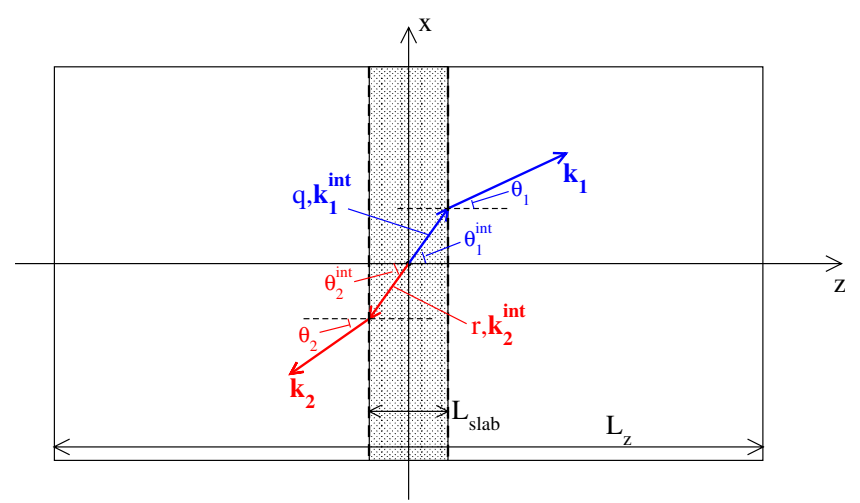

FIG. 4. (Color online) Sketch of the slab geometry under consideration.

$$
\tilde{V}_{r s}(\mathbf{x})=\frac{1}{(2 \pi)^{3}} \int d^{3} \mathbf{k} e^{i \mathbf{k} \cdot \mathbf{x}} V_{r s, k}
$$

does not depend on the integration volume $\mathcal{V}$ and, under reasonable smoothness assumptions for $V_{r s, k}$, is a quite localized function around $\mathbf{x}=0$.

\section{A. Slab geometry}

The advantage of the real-space Hamiltonian (57) is that it is not limited to bulk systems, but can be also applied to finite geometries. For the sake of simplicity, we shall limit ourselves to the plane-parallel slab geometry of Fig. 4. The size $L_{z}$ of the integration box in the $z$ direction orthogonal to the slab plane is assumed to be much longer than the slab thickness $L_{\text {slab }}$. In the transverse $x, y$ directions, the slab is assumed to fill the whole integration box of size $L_{x, y}$.

In order to apply the Fermi golden rule, one must identify the final states of the process. In the present case, they consist of pairs of radiative photons emitted in the empty space surrounding the atomic system. These are created inside the system as polaritons, and are converted into free-space photons when traversing the interface to the external vacuum. For the sake of simplicity, we shall assume that interface reflections are negligible at the frequencies of interest, so that the internal polariton is adiabatically transformed into the photon state at exactly the same energy. As light is collected far outside the slab, we do not have to consider here the case of light being guided inside the slab by total internal reflection.

As usual in scattering problems, the final eigenstates are labeled by the wave vector $\mathbf{k}$ outside the atomic system and their frequency is fixed by the free-space dispersion $\omega=c k$. Outside the Mott insulator, polaritons reduce in fact to photons. Their wave function is the plane wave,

$$
\psi_{\mathbf{k}}^{\text {out }}(\mathbf{x})=\frac{1}{\sqrt{\mathcal{V}}} e^{i \mathbf{k} \cdot \mathbf{x}}
$$

Inside the system, the same eigenstate corresponds to a polariton plane wave of the form [60]

$$
\psi_{\mathbf{k}}^{\mathrm{in}}(\mathbf{x}, r)=\frac{A}{\sqrt{\mathcal{V}}} \delta_{r, \bar{r}} e^{i \mathbf{k}^{\mathrm{int}} \cdot \mathbf{x}} .
$$

The wave vector $\mathbf{k}^{\text {int }}$ and the branch index $\bar{r}$ are fixed by energy conservation in the refraction process at the interface separating the atomic system and the surrounding vacuum: The translational symmetry of the system along the $x, y$ inplane directions guarantees that the $x, y$ components of the wave vector are conserved $k_{x, y}=k_{x, y}^{\text {int }}$, while the band index $\bar{r}$ and the $z$ component $k_{z}^{\text {int }}$ are fixed by the energy conservation $\omega_{\bar{r}, k^{\text {int }}}=c k[61]$.

The amplitude $A$ depends on the external wave vector $\mathbf{k}$ and is fixed by the particle flux conservation condition. As interface reflections are assumed to be negligible, this reads as

$$
c \cos (\theta)=|A|^{2} v_{\bar{r}, k^{\text {int }}}^{g r} \cos \left(\theta^{\text {int }}\right),
$$

where $\theta\left(\theta^{\text {int }}\right)$ are the angles between the $z$ axis and the propagation direction outside (inside) the slab: Note the dramatic increase of the polariton amplitude inside the slab for $v^{g r} \ll c$, a well-known effect in the theory of slow-light propagation [31]. Provided the slab is much thinner than the integration box $L_{z} \gg L_{\text {slab }}$, it is important to remind that the normalization and the density of states do not depend on the slab size, and only involve the total volume $\mathcal{V}=L_{x} L_{y} L_{z}$.

Inserting the explicit form of the polariton wave function $\psi_{\mathbf{k}}(\mathbf{x}, r)$ into the parametric Hamiltonian (57) and using the Fermi golden rule, one immediately gets to an expression for the number of photons emitted into the external vacuum per unit time $d t$, unit surface $d \Sigma=d x d y$, and unit phase-space volume of transverse momentum $d^{2} k_{\perp}=d k_{x} d k_{y}$,

$$
\frac{d N}{d t d \Sigma d^{2} k_{\perp}}=\frac{1}{(2 \pi)^{2}}\left|V_{\overline{r s}, \bar{k}^{\mathrm{int}}}\right|^{2} \frac{L_{\text {slab }}}{\cos \theta^{\mathrm{int}}\left(v_{\bar{r}, k^{\mathrm{int}}}^{g r}+v_{\bar{s}, k^{\mathrm{int}}}^{g r}\right)} .
$$

For each value of the modulation frequency $\omega$, the internal wave vector $\bar{k}^{\text {int }}$ and the branch indices $\bar{r}, \bar{s}$ are selected by the resonance condition (46) with $\mathbf{k}$ replaced by $\mathbf{k}^{\text {int }}$. Inside the system the wave vectors of the emitted polariton pair differ in fact from perfect antiparallelism by a negligible amount $\Delta k_{z} \propto 1 / L_{\text {slab }}$, still their frequencies $\omega_{(\bar{r}, \bar{s}), \bar{k} \text { int }}$ and their group velocities $\mathrm{v}_{(\bar{r}, \bar{s}), k^{\text {int }}}^{g r}$ can be significantly different as soon as distinct branches are considered $\bar{r} \neq \bar{s}$.

It is interesting to note that the result (62) is in perfect agreement with the emission rate predicted in Eq. (49) for a bulk system of volume $\mathcal{V}_{\text {slab }}=L_{x} L_{y} L_{\text {slab }}$ : In the absence of losses, all the polaritons created in the finite slab are in fact emitted from the system as radiation.

By means of a change of variables, the emission rate (62) can be rewritten in its final form as an emission rate per unit surface and unit solid angle $d \Omega$,

$$
\frac{d N_{ \pm}}{d t d \Sigma d \Omega}=\frac{1}{(2 \pi)^{2}}\left|V_{\overline{r s}, k^{\mathrm{int}}}\right|^{2}\left(\frac{\omega_{ \pm}}{c}\right)^{2} \frac{\cos \theta_{ \pm}}{\cos \theta^{\mathrm{int}}} \frac{L_{\mathrm{slab}}}{v_{\bar{r}, \bar{k}^{\mathrm{int}}}^{g r}+v_{\bar{s}, k^{\mathrm{int}}}^{g r}},
$$

where the \pm index, respectively, refer to the photon which is emitted from the slab in the positive $(+)$ or negative $(-) \hat{z}$ 
direction at angles, respectively, $\theta_{ \pm}$with the normal. This

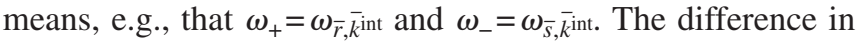
the angular emission density in the \pm directions is due to refraction effects of the $\bar{r}, \bar{s}$ polaritons at the system-vacuum interfaces.

\section{B. Resonant enhancement in a cavity}

A possible way to further enhance the emission intensity is to surround the slab with a pair of mirrors of good reflectivity $R \leqq 1$ : If the modulation frequency $\omega$ is on resonance with a pair of cavity modes, the dynamical Casimir emission results increased by a factor proportional to the finesse of the cavity [21].

For simplicity, let us consider a plane-parallel mirror geometry, so that the cavity modes are labeled by the in-plane component $\mathbf{k}_{\perp}$ of the wave vector, the branch index $\bar{r}$, as well as by a positive integer number $M$ defining the mode order along $z$. Assuming for simplicity that the mirrors are metallic and that no vacuum space is left between the slab and the mirrors, the polaritonic cavity modes are defined by the round-trip quantization condition

$$
k_{z}^{\text {int }} L_{\text {slab }}=\pi M,
$$

and their frequency is equal to the bulk polariton dispersion $\omega_{\bar{r}, k^{\text {int }}}$ at the relevant wave vector $k^{\text {int }}$. Because of the nontrivial shape of the dispersion law $\omega_{\bar{r}, k}$ int as a function of $k^{\text {int }}$, the cavity modes are not equally spaced in frequency. Their polaritonic wave function has a simple sinusoidal form (the mirrors are at $\left.z=0, L_{\text {slab }}\right)$,

$$
\psi_{\bar{r}, M, \mathbf{k}_{\perp}}(\mathbf{x}, r)=\sqrt{\frac{2}{L_{\mathrm{slab}} L_{x} L_{y}}} \sin \left(\frac{\pi M z}{L_{\mathrm{slab}}}\right) \delta_{r, \bar{r}} e^{i \mathbf{k}_{\perp} \cdot \mathbf{x}},
$$

and their radiative decay rate into externally propagating photons is equal to

$$
\gamma=\frac{1-R}{L_{\text {slab }}} \cos \left(\theta^{\text {int }}\right) v_{\bar{r}, k^{\text {int }}}^{g r} .
$$

As expected, the slower the group velocity $v^{g r}$, the smaller the decay rate $\gamma$.

Fermi golden rule can again be used to estimate the emission rate in the cavity geometry. As final states, pairs of cavity photons must be considered, with a finite linewidth equal to $\gamma[35]$. For a thick enough cavity, the mode order at the frequency of interest is $M \gg 1$ and overlap factor strongly privileges polariton emission into pairs of cavity modes of the same order $M$; the efficiency of all other processes is suppressed by their spatial phase mismatch. The emission rate into a pair of such modes is then easily obtained,

$$
\frac{d N}{d t d \Sigma d^{2} k_{\perp}}=\frac{1}{\pi^{2}}\left|V_{\overline{r s}, \bar{k}^{\text {int }}}\right|^{2} \frac{\gamma_{T}}{\left(\omega-\omega_{T}\right)^{2}+\frac{\gamma_{T}^{2}}{4}} \stackrel{\omega=\omega_{T}}{\rightarrow} \frac{1}{\pi^{2} \gamma_{T}}\left|V_{\overline{r s}, \bar{k} \bar{k}^{\mathrm{int}}}\right|^{2},
$$

where $\omega_{T}$ and $\gamma_{T}$ are here the sum of, respectively, the frequencies and the linewidths of the pair of modes under examination.
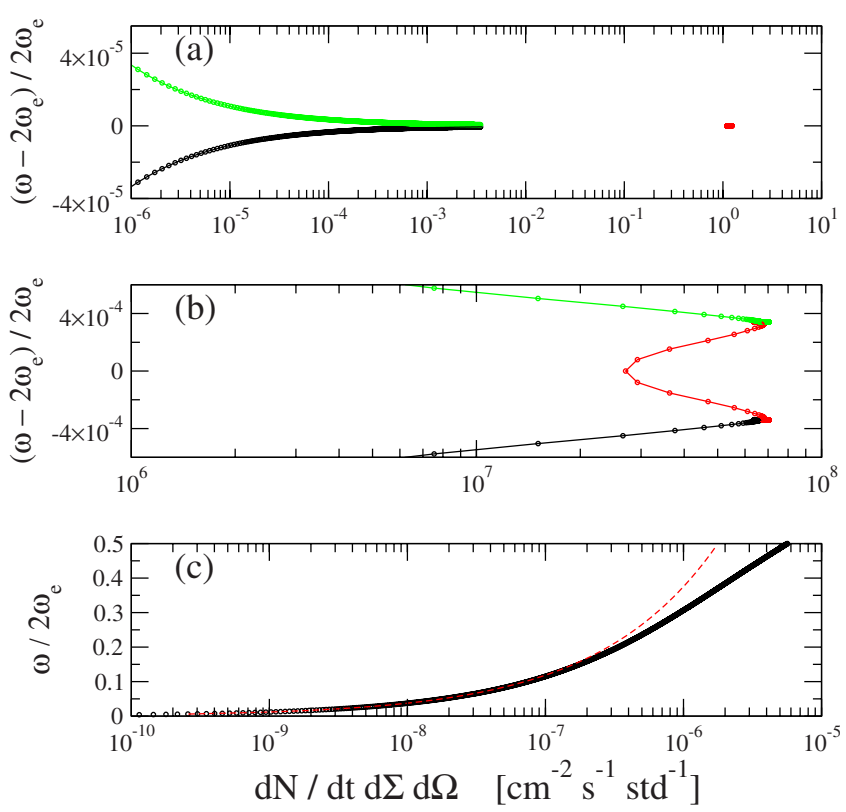

FIG. 5. (Color online) Spectrum of the dynamical Casimir emission rate per unit surface and unit solid angle around the normal direction $\left(\theta_{ \pm}=0\right)$ as a function of the modulation frequency $\omega$ for the resonant case $\omega_{e}=\widetilde{\omega}_{m}$, a slab thickness $L_{\text {slab }}=10 \mu \mathrm{m}$ in the absence of enclosing cavity, and a relative modulation amplitude $\delta \Omega_{C} / \Omega_{C}=0.05$. Same system parameters as in Fig. 3. (a) Weak dressing $\Omega_{C} / \bar{C}=1.86 \cdot 10^{-4} \ll 1 ;\left(\right.$ b,c) strong dressing $\Omega_{C} / \bar{C}=2$. The circles in $(a, b, c)$ are the result of the exact calculation (63). The solid lines in $(\mathrm{a}, \mathrm{b})$ are the analytical approximation (52). The red dashed line in (c) is the prediction of the dispersionless, timevarying dielectric model based on Eq. (55). Panels (a,b) correspond to the band dispersion and absorption spectra shown in Figs. 3(a)-3(d). Black, red, and green colors in (a,b) refer to the lower (LP), the middle (MP) and the upper (UP) polaritons, respectively.

By comparing Eq. (67) with the result (62) in the absence of the enclosing cavity, and using Eq. (66), it is immediate to see that the emission rate for an excitation exactly on resonance with a pair of modes $\left(\omega=\omega_{T}\right)$ is enhanced by a factor $4 /(1-R)$. This enhancement effect is quite general and holds for a variety of optical processes [5]; for well reflecting mirrors $R \leqq 1$, it can be quite dramatic.

\section{QUANTITATIVE DISCUSSION AND EXPERIMENTAL CONSIDERATIONS}

In the previous sections, we have obtained simple analytical expressions relating the emission intensity in the different geometries to the $V_{r s, k}$ parameter (50) which carries information on the microscopic optical properties of the atomic medium. In the present section, we shall conclude the study by providing quantitative estimation of the emission intensity for realistic systems, and discussing the most relevant issues that are likely to arise in the design of an actual experiment.

An example of emission intensity spectrum is shown in Fig. 5 for a $L_{\text {slab }}=10 \mu \mathrm{m}$ slab of Rb atomic Mott insulator in the absence of cavity as described by Eq. (63). The coupling amplitude $\Omega_{C}$ is assumed to have the periodic time depen- 


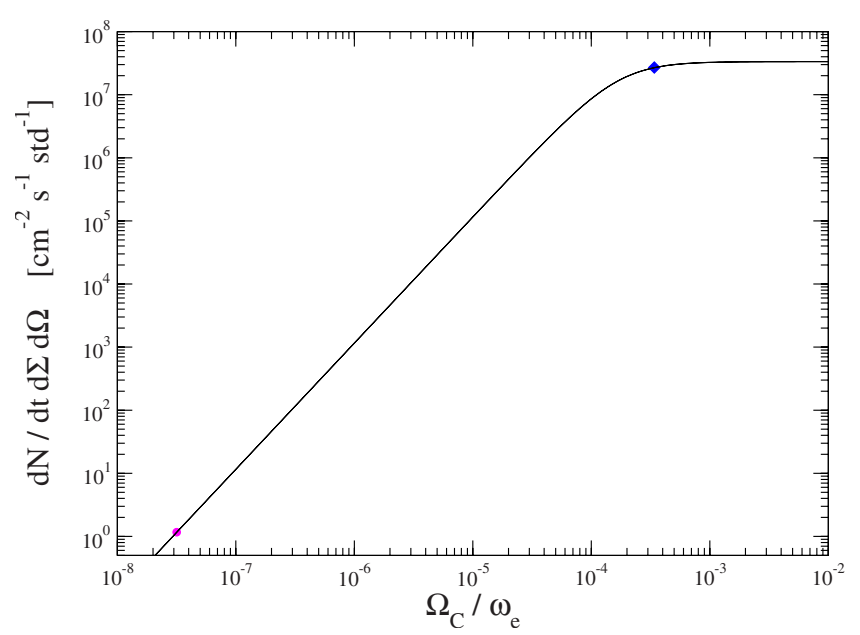

FIG. 6. (Color online) Prediction (63) for the dynamical Casimir emission rate per unit surface and unit solid angle around the normal direction $\left(\theta_{ \pm}=0\right)$ as a function of the coupling amplitude $\Omega_{C}$ for a fixed value of the relative modulation amplitude $\delta \Omega_{C} / \Omega_{C}$ $=0.05$ at the fully resonant point $\omega / 2=\omega_{e}=\widetilde{\omega}_{m}$. Same system parameters as in Fig. 5: The magenta circle and the blue diamond correspond to, respectively, the weak (a) and strong (b) dressing regimes.

dence (51) at a frequency $\omega$, while its frequency $\omega_{C}$ is kept fixed: the modulation of $\Omega_{C}$ then consists of a pair of coherent sidebands at $\pm \omega$ around the carrier frequency $\omega_{C}$ $=\left|\omega_{e}-\omega_{m}\right|$. As expected, the central MP polariton branch appears as the most favorable region thanks to the combination of reduced group velocity and significant resonant mixing of light and matter excitations. In panels (a) and (b) the result of the complete calculation (circles) is compared with the analytical approximation (52) (solid line): The agreement is excellent throughout the whole resonance region. In panel (c), the same calculation is performed in the low-frequency region and is compared to Law's result (55) for a dispersionless medium [44]: Inserting in Eq. (55) the refractive index variation that follows from time dependence of $\Omega_{C}$ and using the general formula (63) for the emission rate, one obtains the dashed curve in Fig. 6(c). As expected, at very low frequencies the agreement is excellent, but dispersion effects start playing a significant role already for $\omega \approx 0.2 \omega_{e}$.

All of the panels in Fig. 5 have been calculated for the same value of the modulation amplitude $\delta \Omega_{C} / \Omega_{C}$ : in spite of the higher value of $v^{g r}$, a larger value of $\Omega_{C}$ is favorable in view of maximizing the emission intensity as it allows for a stronger modulation of the optical properties. A specific plot of the resonance emission rate as a function of the coupling amplitude $\Omega_{C}$ is shown in Fig. 6: As predicted in Sec. IV B, the Casimir emission first grows as $\Omega_{C}^{2}$ for $\Omega_{C} \ll \bar{C}$, then saturates to a finite value for $\Omega_{C} \gg \bar{C}$.

Remarkably, the emission intensity at resonance can reach quite substantial values already in the absence of a cavity. In the weak dressing case $\left(\Omega_{C} / 2 \pi=12 \mathrm{MHz}\right)$, the rate of emitted photons from a $1 \mathrm{~cm}^{2}$ system in the unit solid angle around the normal is of the order of 1 photon per second [see Fig. 5(a)]. Then it quadratically (see Fig. 6) increases for growing $\Omega_{C}$, to eventually saturate around a value larger than
$10^{7}$ photons per second for a huge dressing $\Omega_{C} / 2 \pi$ $\simeq 100 \mathrm{GHz}$ [see Fig. 5(b)]. For alkali-metal atoms such as $\mathrm{Rb}$, a dressing amplitude $\Omega_{C} / 2 \pi$ in the $10 \mathrm{MHz}$ range corresponds to intensities of the dressing beam in the $\mathrm{mW} / \mathrm{cm}^{2}$ range [40].

A crucial difficulty of most dynamical Casimir experiments consists of varying the optical properties of the system at a high enough speed. In our specific setup, this amounts to modulating the dressing beam at a frequency which is resonant with the creation of a pair of MP.

Although this is hardly done with the almost symmetric $\Lambda$ schemes currently used in slow-light experiments with alkali-metal atom samples $[12,15,16]$, still strongly asymmetric $\Lambda$ schemes as shown in Fig. 1(c) can be used. The ground state of the atom is now the $m$ state, while the $g$ state is a long-lived, high-energy metastable state. The dressing beam then acts on the $m \rightarrow e$ transition at a frequency $\left|\omega_{e}-\omega_{m}\right|$ higher than 2 times the frequency $\left|\omega_{e}-\omega_{g}\right|$ of the $g \rightarrow e$ transition on which the dynamical Casimir radiation is to be emitted: No principle difficulties then appear to prevent one from modulating the dressing amplitude $\Omega_{C}$ at the required frequency $\omega \simeq 2\left|\omega_{e}-\omega_{g}\right|$. This can be obtained, e.g., by mixing the carrier at $\omega_{C}$ with another beam at $\omega$ on a suitable nonlinear crystal.

Strongly asymmetric $\Lambda$ configurations can be found, e.g., in alkali-metal-earth atoms [49] whose laser cooling and trapping techniques have experienced remarkable advances in the last few years [50]. A specific choice in view of our dynamical Casimir application can be ${ }^{88} \mathrm{Sr}$ atoms, whose $4 d{ }^{1} D_{2}$ metastable state appears to have the required properties to be used as the $g$ state of a strongly asymmetric $\Lambda$ scheme. It is in fact connected to the excited $5 p{ }^{1} P_{1}$ state by an optically active infrared transition at $\lambda=6.5 \mu \mathrm{m}$ which, for a lattice spacing of $300 \mathrm{~nm}$, gives a value $\bar{C} / \omega_{e} \simeq 5$ $\times 10^{-5}$ not far from the $\mathrm{Rb}$ one. The $5 p{ }^{1} P_{1}$ state can be dressed by driving the atom on the $\lambda=461 \mathrm{~nm}$ transition from the absolute ground state of the atom $5 s^{2}{ }^{1} S_{0}$ $\rightarrow 5 p{ }^{1} P_{1}$. Before performing the dynamical Casimir experiment, atoms must be optically pumped in the $4 d{ }^{1} D_{2}$ metastable state, e.g., by means of a $\pi$ Raman pulse; the lifetime of the state being of $0.33 \mathrm{~ms}$, there is enough time left to carry out the dynamical Casimir experiment before atoms decay to the ground state via the $5 p{ }^{3} P_{J}$ state: Even for a group velocity as low as $10 \mathrm{~m} / \mathrm{s}$, the transit time across a $10-\mu \mathrm{m}$-thick cloud is in fact much shorter, of the order of $1 \mu$ s. Note also that this spontaneous decay channel involves photons at $1.8 \mu \mathrm{m}$ and $689 \mathrm{~nm}$, in completely different spectral regions from the dynamical Casimir ones at $6.5 \mu \mathrm{m}$, which can therefore be spectrally isolated with no difficulty.

Another possible simpler solution is to stick to alkalimetal atoms such as Rb, but use a ladder scheme [see Fig. 1(b)] where the $m$ state is a electronically highly excited state instead of a $\Lambda$ one. Even though the unavoidable radiative contribution to $\gamma_{m}$ prevents the EIT effect from completely killing the absorption, still DCE light can escape the slab without dramatic losses. This, at least for the relatively large values of $\Omega_{C}$ that appear to be the most favorable for the observation of DCE [Fig. 2(d)]. 
Although from a rigorous standpoint this goes beyond the Fano-Hopfield model under investigation here, still the conclusions of our analysis suggest that the DCE experiment might be performed with less demanding atomic samples, e.g., Bose-Einstein condensates or even thermal gases. For the high value of $\Omega_{C}$ that has been identified as the most favorable regime, the consequences of spontaneous emission from both the $e$ and the $m$ states do not appear in fact to be dramatic. Additional difficulties could however arise from the inhomogeneous broadening of $\widetilde{\omega}_{m}$ due to the spatial variations of the trapping and interaction potentials, and from the reduced value of the atomic density (and then of the light-matter coupling coefficient $\bar{C}$ ). While this latter effect can be a serious issue in nondegenerate clouds, the inhomogeneous broadening of $\widetilde{\omega}_{m}$ appears again to be easily overcome by the strong dressing amplitude $\Omega_{C}$. A complete discussion of these issues will be the subject of forthcoming work.

\section{CONCLUSIONS}

In this paper we have performed a systematic analysis of the optical properties of a gas of coherently dressed threelevel atoms trapped in an optical lattice in a Mott insulator state. The extreme degree of coherence of this system allows for propagation of light at ultraslow group velocities for long times and distances. The optical properties of the medium can be controlled in real time by varying the amplitude and the frequency of the dressing field. For sufficiently fast modulation rates, the zero-point fluctuations of the polariton vacuum state are converted into observable radiation by dynamical Casimir effect.

We have developed a general theory to quantitatively characterize the dynamical Casimir emission in terms of a simple parametric Hamiltonian and we have identified the most favorable case of a resonant dressing frequency whose amplitude is periodically modulated in time. Experimentally realistic geometries such as plane-parallel slabs and planar cavities are analyzed in detail. Remarkably, a sizeable radiation intensity is predicted for state-of-the-art systems and no spurious emission from blackbody radiation or incoherent luminescence is expected to mask the dynamical Casimir signal.

\section{ACKNOWLEDGMENTS}

We are grateful to G. C. La Rocca, M. Artoni, R. Sturani, G. Ferrari, C. Tozzo, F. Dalfovo, Y. Castin for stimulating discussions. I.C. acknowledges hospitality at the Institut Henri Poincare-Centre Emile Borel and financial support from CNRS. FB acknowledges hospitality at the Institut Henri Poincare-Centre Emile Borel and financial support from ESF-QUDEDIS network through Short Visit Grant, No. 1802.
[1] Confined Electrons and Photons, edited by E. Burstein and C. Weisbuch (Plenum, New York, 1995).

[2] Confined Photon Systems, edited by H. Benisty, J.-M. Gérard, R. Houdré, and C. Weisbuch (Springer, Heidelberg, 1999).

[3] Cavity Quantum Electrodynamics, edited by P. R. Berman (Academic, Boston, 1994).

[4] D. Dini, R. Kohler, A. Tredicucci, G. Biasiol, and L. Sorba, Phys. Rev. Lett. 90, 116401 (2003); E. Dupont, H. C. Liu, A. J. SpringThorpe, W. Lai, and M. Extavour, Phys. Rev. B 68, 245320 (2003); A. A. Anappara, A. Tredicucci, G. Biasiol, and L. Sorba, Appl. Phys. Lett. 87, 051105 (2005); R. Colombelli, C. Ciuti, Y. Chassagneux, and C. Sirtori, Semicond. Sci. Technol. 20, 985 (2005).

[5] V. Berger, J. Opt. Soc. Am. B 14, 1351 (1997); A. Fainstein and B. Jusserand, Phys. Rev. B 57, 2402 (1998); G. Ferrari and I. Carusotto, J. Opt. Soc. Am. B 22, 2115 (2005).

[6] M. Greiner, O. Mandel, T. Esslinger, T. W. Hänsch, and I. Bloch, Nature (London) 415, 39 (2002).

[7] M. P. A. Fisher, P. B. Weichman, G. Grinstein, and D. S. Fisher, Phys. Rev. B 40, 546 (1989); D. Jaksch, C. Bruder, J. I. Cirac, C. W. Gardiner, and P. Zoller, Phys. Rev. Lett. 81, 3108 (1998).

[8] M. Köhl, H. Moritz, T. Stöferle, K. Günter, and T. Esslinger, Phys. Rev. Lett. 94, 080403 (2005).

[9] U. Fano, Phys. Rev. 103, 1202 (1956); J. J. Hopfield, Phys. Rev. 112, 1555 (1958).

[10] Y. D. Chong, D. E. Pritchard, and M. Soljacić, Phys. Rev. B 75, 235124 (2007).
[11] H. Zoubi and H. Ritsch, Phys. Rev. A 76, 013817 (2007).

[12] E. Arimondo, in Progress in Optics $X X X V$, edited by E. Wolf (Elsevier Science, New York, 1996), p. 257.

[13] M. Fleischhauer, A. Imamoglu, and J. P. Marangos, Rev. Mod. Phys. 77, 633 (2005).

[14] A. B. Matsko et al., Adv. At., Mol., Opt. Phys. 46, 191 (2001).

[15] L. V. Hau, S. E. Harris, Z. Dutton, and C. H. Behroozi, Nature (London) 397, 594 (1999); S. Inouye, R. F. Low, S. Gupta, T. Pfau, A. Gorlitz, T. L. Gustavson, D. E. Pritchard, and W. Ketterle, Phys. Rev. Lett. 85, 4225 (2000); M. M. Kash, V. A. Sautenkov, A. S. Zibrov, L. Hollberg, G. R. Welch, M. D. Lukin, Y. Rostovtsev, E. S. Fry, and M. O. Scully, ibid. 82, 5229 (1999); D. Budker, D. F. Kimball, S. M. Rochester, and V. V. Yashchuk, ibid. 83, 1767 (1999).

[16] D. F. Phillips, A. Fleischhauer, A. Mair, R. L. Walsworth, and M. D. Lukin, Phys. Rev. Lett. 86, 783 (2001); C. Liu, Z. Dutton, C. H. Behroozi, and L. V. Hau, Nature (London) 409, 490 (2001).

[17] H. B. G. Casimir, Proc. K. Ned. Akad. Wet. 51, 793 (1948); I. E. Dzyaloshinskii, E. M. Lifshitz, and L. P. Pitaevskii, Adv. Phys. 10, 165 (1961).

[18] M. J. Sparnaay, Physica 24, 751 (1958); I. I. Abrikosova and B. V. Deriagin, Sov. Phys. JETP 4, 1957 (1957); S. K. Lamoreaux, Phys. Rev. Lett. 78, 5 (1997); U. Mohideen and A. Roy, ibid. 81, 4549 (1998); H. B. Chan, V. A. Aksyuk, R. N. Kleiman, D. J. Bishop, and F. Capasso, Science 291, 1941 (2001); T. Ederth, Phys. Rev. A 62, 062104 (2000); G. Bressi, G. Carugno, R. Onofrio, and G. Ruoso, Phys. Rev. Lett. 88, 
041804 (2002). Reviews can be found in, e.g., A. Bordag, U. Mohideen, and V. M. Mostepanenko, Phys. Rep. 353, 1 (2001); S. K. Lamoreaux, Rep. Prog. Phys. 68, 201 (2005).

[19] G. T. Moore, J. Math. Phys. 11, 2679 (1970); S. A. Fulling and P. C. W. Davies, Proc. R. Soc. London, Ser. A 348, 393 (1976); P. C. W. Davies and S. A. Fulling, ibid. 356, 237 (1977).

[20] M. Kardar and R. Golestanian, Rev. Mod. Phys. 71, 1233 (1999).

[21] A. Lambrecht, M.-T. Jaekel, and S. Reynaud, Phys. Rev. Lett. 77, 615 (1996); M.-T. Jaekel and S. Reynaud, Rep. Prog. Phys. 60, 863 (1997); A. Lambrecht, J. Opt. B: Quantum Semiclassical Opt. 7, S3 (2005).

[22] V. V. Dodonov, A. B. Klimov, and D. E. Nikonov, Phys. Rev. A 47, 4422 (1993); E. Yablonovitch, Phys. Rev. Lett. 62, 1742 (1989).

[23] Y. E. Lozovik, V. G. Tsvetus, and E. A. Vinogradov, Phys. Scr. 52, 184 (1995); JETP Lett. 61, 723 (1995); M. Crocce, D. A. R. Dalvit, F. C. Lombardo, and F. D. Mazzitelli, Phys. Rev. A 70, 033811 (2004).

[24] C. Braggio, G. Bressi, G. Carugno, C. Del Noce, G. Galeazzi, A. Lombardi, A. Palmieri, G. Ruoso, and D. Zanello, Europhys. Lett. 70, 754 (2005); W.-J. Kim, J. H. Brownell, and R. Onofrio, ibid. 78, 21002 (2007); C. Braggio, G. Bressi, G. Carugno, C. Del Noce, G. Galeazzi, A. Lombardi, A. Palmieri, G. Ruoso, and D. Zanello, ibid. 78, 21003 (2007).

[25] F.-X. Dezael, Ph.D. thesis, Université Paris VI, 2007, http:// tel.archives-ouvertes.fr/tel-00165149/

[26] M. O. Scully, V. V. Kocharovsky, A. Belyanin, E. Fry, and F. Capasso, Phys. Rev. Lett. 91, 243004 (2003); A. Belyanin, V. V. Kocharovsky, F. Capasso, E. Fry, M. S. Zubairy, and M. O. Scully, Phys. Rev. A 74, 023807 (2006); W.-J. Kim, J. H. Brownell, and R. Onofrio, Phys. Rev. Lett. 96, 200402 (2006).

[27] P. Agostini and L. F. DiMauro, Rep. Prog. Phys. 67, 813 (2004).

[28] H. Saito and H. Hyuga, Phys. Rev. A 65, 053804 (2002).

[29] C. Ciuti, G. Bastard, and I. Carusotto, Phys. Rev. B 72, 115303 (2005); C. Ciuti and I. Carusotto, Phys. Rev. A 74, 033811 (2006).

[30] S. De Liberato, C. Ciuti, and I. Carusotto, Phys. Rev. Lett. 98, 103602 (2007).

[31] M. Fleischhauer and M. D. Lukin, Phys. Rev. Lett. 84, 5094 (2000); M. Fleischhauer and M. D. Lukin, Phys. Rev. A 65, 022314 (2002).

[32] I. Bloch, J. Dalibard, and W. Zwerger e-print arXiv:0704.3011, Rev. Mod. Phys. (to be published); O. Morsch and M. Oberthaler, ibid. 78, 179 (2006).

[33] A discussion of the absorptive photonic band-gap crystals in the presence of a sinusoidal spatial modulation of $\Omega_{C}(\mathbf{R})$ can be found in M. Artoni and G. C. La Rocca, Phys. Rev. Lett. 96, 073905 (2006).

[34] C. S. Adams and E. Riis, Prog. Quantum Electron. 21, 1 (1997).

[35] C. Cohen-Tannoudji, J. Dupont-Roc, and G. Grynberg, AtomPhoton Interactions. Basic Processes and Applications (Wiley, New York, 1998).

[36] F. Bariani and I. Carusotto, J. Eur. Opt. Soc. Rapid Publ. 3, 08005 (2008).

[37] See, e.g., V. M. Agranovich and B. S. Toschich, Sov. Phys. JETP 26, 104 (1968).
[38] J. D. Jackson, Classical Electrodynamics, 2nd ed. (Wiley, New York, 1975).

[39] G. S. Agarwal and R. W. Boyd, Phys. Rev. A 60, R2681 (1999).

[40] See, e.g., D. A. Steck, Alkali D line data, available at http:// steck.us/alkalidata/

[41] I. Carusotto, M. Artoni, and G. C. La Rocca, JETP Lett. 72, 289 (2000).

[42] W. C. Tait, Phys. Rev. B 5, 648 (1972).

[43] H. A. Macleod, Thin-Film Optical Filters (CRC, Boca Raton, FL, 2001).

[44] C. K. Law, Phys. Rev. A 49, 433 (1994).

[45] M. Artoni, A. Bulatov, and J. Birman, Phys. Rev. A 53, 1031 (1996).

[46] T. Stöferle, H. Moritz, C. Schori, M. Köhl, and T. Esslinger, Phys. Rev. Lett. 92, 130403 (2004); C. Tozzo, M. Krämer, and F. Dalfovo, Phys. Rev. A 72, 023613 (2005); M. Krämer, C. Tozzo, and F. Dalfovo, ibid. 71, 061602(R) (2005).

[47] D. F. Walls and G. J. Milburn, Quantum Optics (SpringerVerlag, Berlin, 1994).

[48] S. I. Pekar, Zh. Eksp. Teor. Fiz. 33, 1022 (1958) [Sov. Phys. JETP 6, 785 (1958)]; H. C. Schneider, F. Jahnke, S. W. Koch, J. Tignon, T. Hasche, and D. S. Chemla, Phys. Rev. B 63, 045202 (2001).

[49] H. G. Kuhn, Atomic Spectra (Academic, New York, 1969).

[50] S. B. Nagel, C. E. Simien, S. Laha, P. Gupta, V. S. Ashoka, and T. C. Killian, Phys. Rev. A 67, 011401(R) (2003); Y. Takasu, K. Maki, K. Komori, T. Takano, K. Honda, M. Kumakura, T. Yabuzaki, and Y. Takahashi, Phys. Rev. Lett. 91, 040404 (2003); F. Sorrentino, G. Ferrari, N. Poli, R. Drullinger, and G. M. Tino, Mod. Phys. Lett. B 20, 1287 (2006).

[51] The main consequence of including nonresonant higher bands is a small shift of the bands which generalizes the Lamb shift [35] to the present case of a system of many atoms in a lattice geometry. A discussion of these features is postponed to a future work where all the related ultraviolet divergence issues will be developed in full detail. A brief discussion of the polariton bands in the case when $\omega_{e} a_{L} / c \simeq 1$ and Bragg scattering processes are important can be found in [36] and in the references cited therein.

[52] The commutator of raising $a_{e, j}^{\dagger}=|e\rangle_{j}\left\langle\left. g\right|_{j}\right.$ and lowering $a_{e, j}$ $=|g\rangle_{j}\left\langle\left. e\right|_{j}\right.$ operators acting on the same atom is $\left[a_{e, j}, a_{e, j}^{\dagger}\right]$ $=|g\rangle_{j}\left\langle\left. g\right|_{j}-\mid e\right\rangle_{j}\left\langle\left. e\right|_{j}=\mathbf{1}_{j}-\mid m\right\rangle_{j}\left\langle\left. m\right|_{j}-2 \mid e\right\rangle_{j}\left\langle\left. e\right|_{j}\right.$, where $\mathbf{1}_{j}$ is the identity operator on the $j$ th atom. Operators acting on different atoms commute. The commutator of the collective atomic operators defined in Eq. (8) has the form $\left[\hat{a}_{\mathbf{k}}, \hat{a}_{\mathbf{k}^{\prime}}^{\dagger}\right]=\delta_{\mathbf{k}, \mathbf{k}^{\prime}}$

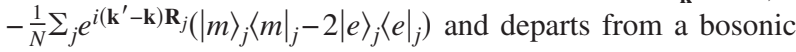
one by terms proportional to the total number of excitations present in the system over the total number of atoms [37]

[53] Coupling to the longitudinal e.m. field corresponds to the static dipole-dipole Coulomb interaction $[9,35]$. At low excitation regimes, this term is only responsible for a slight redshift of the $g \rightarrow e$ transition frequency $\omega_{e}$ by $\Delta \omega_{e}=-2 \bar{C}^{2} /\left(3 \omega_{e}\right)$. For the typical values of the systems under examination here, this shift is on the order of a few $10 \mathrm{MHz}$ and can be reincorporated in the definition of $\omega_{e}$. In a semiclassical description of light-matter interaction, such a shift naturally appears when the Clausius-Mossotti form of the dielectric constant is used [38,39].

[54] Though small, this term is crucial to preserve gauge invariance 
and avoid unphysical behaviors in the $k \rightarrow 0$ limit.

[55] In a deep lattice, the branching ratio for the decay of $e$ into motional states other than the ground state is equal to $\eta=1$ $-\exp \left(-E_{R} / 2 \hbar \omega_{\mathrm{HO}}\right) \simeq 1 / \sqrt{16 s}$, where $s=V_{0} / E_{R}$ and $\omega_{\mathrm{HO}}$ is the frequency for quasiharmonic oscillations around the equilibrium position at each lattice site [32]. As expected, $\eta \rightarrow 0$ in the strong lattice limit $s \gg 0$. Including processes beyond the Lamb-Dicke limit gives a contribution to $\gamma_{e}$ equal to $\eta \gamma_{e \rightarrow g}$, where $\gamma_{e \rightarrow g}$ is the $e \rightarrow g$ radiative decay rate of an isolated atom in free space.

[56] In our formalism, such processes correspond to the conversion of a $e$ excitation into a $m$ excitation plus a polariton at a frequency $\omega_{e}-\omega_{m}$ far from the resonance region $\omega_{e}-\omega_{g}$. Using Fermi golden rule, it is immediate to see that the corresponding decay rate is indeed equal to the free space spontaneous radiative decay rate for the $e \rightarrow m$ transition.

[57] Note that this plot does not include the extinction effects that occur for frequencies within the (very small) gaps between the bands, and which are, e.g., responsible for the operation of distributed Bragg reflectors $[1,43]$.

[58] For the $\mathrm{Rb}$ atoms under consideration here, an absorption length of $1 \mathrm{~m}$ corresponds to $\kappa^{\mathrm{abs}} / k_{e}=1.24 \times 10^{-7}$.

[59] A similar perturbative calculation for a periodic modulation of $\widetilde{\omega}_{m}$ would lead to a vanishing result at the lowest order in perturbation theory. This is a direct consequence of the vanishing value of $v_{r, k}^{m, 1}$ obtained in Eq. (36). A complete calculation including next-order terms results in a dynamical Casimir emission intensity orders of magnitude weaker than for a modulation of $\Omega_{C}$.

[60] In real space, the polariton wave function $\psi_{\mathbf{k}}^{\text {in }}(\mathbf{x}, r)$ depends in fact on the spatial coordinate $\mathbf{x}$ and on the branch index $r$. This latter can be interpreted as a sort of internal degree of freedomlike spin.

[61] As the bands have no spectral overlap, at most one propagating polariton mode exists for a given frequency $\omega$ : This guarantees that no additional boundary conditions are required here [48]. Rather, the energy conservation condition can be satisfied only outside the (very small) gaps that open in the polariton dispersion between the LP and the MP, and between the MP and the UP. For frequencies inside these gaps, the radiative eigenstates are confined in the external vacuum and cannot penetrate the atomic system: The dynamical Casimir emission is therefore strongly suppressed. On the other hand, light escape from the atomic system is possible only for polariton modes which lie within the so-called radiative cone $k_{x}^{2}+k_{y}^{2} \leq \omega^{2} / c^{2}$ : Polaritons which are created outside this cone remain in fact trapped in the slab and propagate through it as in a waveguide. 